\title{
Transcriptomic profiles of Plasmodium falciparum and Plasmodium vivax-infected individuals in Indonesia
}

Katalina Bobowik ${ }^{1,2,3 *}$, Din Syafruddin ${ }^{4,5}$, Chelzie Crenna Darusallam ${ }^{6}$, Herawati Sudoyo 6,7 , Christine Wells ${ }^{3}$, Irene Gallego Romero ${ }^{1,2,3,8 *}$

1 Melbourne Integrative Genomics, University of Melbourne, Royal Parade, 3010, Parkville, Victoria, Australia

2 School of BioSciences, The University of Melbourne, Royal Parade, 3010, Parkville, Australia

3 The Centre for Stem Cell Systems, Faculty of Medicine, Dentistry and Health Sciences, The University of Melbourne, 30 Royal Parade Parkville, Victoria 3010, Australia

4 Department of Parasitology, Faculty of Medicine, Universitas Hasanuddin, Makassar, 90245, Indonesia

5 Malaria and Vector Resistance Laboratory, Eijkman Institute for Molecular Biology, Jakarta, Indonesia

6 Genome Diversity and Diseases Laboratory, Eijkman Institute for Molecular Biology, Jakarta, Indonesia 7 Sydney Medical School, University of Sydney, Sydney, NSW, Australia

8 Center for Genomics, Evolution and Medicine, Institute of Genomics, University of Tartu, Riia 23b, 51010 Tartu, Estonia

* Correspondence: irene.gallego@unimelb.edu, kbobowik@student.unimelb.edu.au

\section{Abstract}

Malaria is one of the leading causes of illness and death globally. The vast majority of transcriptomic studies of the impact of malaria on human hosts have been conducted on populations of African ancestry suffering from Plasmodium falciparum infection, making it unclear whether biological responses observed in these studies can be generalised to other populations. Here, we perform differential expression analysis between healthy controls and malaria-infected patients within Indonesia, a country of over 260 million people which has substantial morbidity due to endemic malaria. We find that in samples infected with P. falciparum and $P$. vivax, there is an upregulation of genes involved in inflammation, the immediate early immune response, translation, and apoptosis. When comparing these findings to transcriptomic studies conducted 
in Africa (on P. falciparum) and South America (on P. vivax), we find that many pathways are shared. This is particularly apparent for receptor recognition and inflammation-related genes in P. falciparum and innate immune and chemokine-related genes in P. vivax infection. However, we also find that many genes are unique to the Indonesian population, particularly those involved in RNA processing, splicing, and cell surface receptor genes. This study provides a more comprehensive view of malaria infection outside of Africa and contributes to a better characterisation of malaria pathogenesis within humans across a range of genetic architectures.

\section{Background}

Malaria, one of the leading causes of illness and death globally [1], has exerted some of the strongest selective pressures on the human genome [2], but little is known about how the human transcriptional response to malaria varies across populations. Transcriptomic studies conducted on the host response to Plasmodium infection have been a critical tool in attempting to explain the functional mechanisms behind malaria pathogenesis, yet the vast majority of transcriptomic studies on the human host have been conducted on populations within Africa [3-5]. It is clear that human adaptation to malaria has occurred locally multiple times [2]. For example, Southeast Asian ovalocytosis, caused by a deletion in the SLC4A1 gene, confers a broad-spectrum protection against multiple malaria parasites and is found in high frequencies throughout Island Southeast Asia and the Malay Peninsula [6] while HbS, or the sickle haemoglobin allele, is found most commonly in Africa and can result in a 10 -fold lower risk of severe malaria $[7,8]$. Therefore, there is a crucial need to understand how the host response varies across different ethnic backgrounds, as these differences can impact disease progression and allow for better diagnosis and therapeutics.

Despite being the second-most affected malaria region in the world [9] and hosting significant levels of human genetic diversity [10], Island Southeast Asia - a region which includes the countries of Brunei, Indonesia, Papua New Guinea, the Philippines, and East Timor, as well as smaller island nations - is nearly unrepresented in transcriptomic studies analysing the human host response to malaria. To our knowledge, only one transcriptomic study analysing human gene expression has been conducted on malaria patients within Island Southeast Asia [11], focusing on understanding the relationship between gene expression in humans and P. falciparum, as well as how gene expression correlates with clinical data such as malaria severity, age, and body temperature.

However, there is still a lack of understanding of how Plasmodium-infected individuals respond to infection compared to healthy controls. Furthermore, P. vivax, which differs in its biology to P. falciparum, 
is also a common cause of infection in Island Southeast Asia and the host response to it is even more poorly understood. In order to address these gaps, here we analyse the transcriptional profile of malaria-infected individuals within Island Southeast Asia. Combining previously collected whole blood RNA-seq data from healthy Indonesian individuals [12], with the dataset generated by Yamagishi et al. [11], we analyse which pathways and genes are differentially regulated between patients with simple forms of malaria (uncomplicated and asymptomatic) and healthy controls. Given the different genetic backdrop and strains of Plasmodium species within Island Southeast Asia, this study aims to characterise the transcriptomic profile of infection in this region, and determine whether it differs when compared to previous studies - predominantly within the continent of Africa. 


\section{Methods}

\section{Samples}

Samples were collated from the only two whole-blood RNA-seq datasets containing malaria-infected and control samples from Indonesia we could find $[11,12]$. We combined samples from these two different datasets for two main reasons: First, both datasets are unbalanced between cases and controls, and if analysed separately this would lead to an overrepresentation of one group (e.g., case or control) over the other. Secondly, collating data from both studies results in more samples and therefore increased power to detect differentially expressed (DE) genes.

The first dataset consists of 100 base-pair, paired-end data from whole blood from 116 male individuals (plus 6 technical replicates) collected by members of the Eijkman Institute on the Indonesian islands of Sumba, Mentawai, and on the Indonesian side of New Guinea Island (raw data are available from the European Genome-phenome Archive study EGAS00001003671; as described in [12]), hereafter referred to as the Natri dataset. All collections followed protocols for the protection of human subjects established by institutional review boards at the Eijkman Institute (EIREC \#90 and \#126); the analyses in this publication were additionally approved by University of Melbourne's Human Ethics Advisory Group (1851585.1).

The second dataset consists of 36 base-pair, single-end data from whole blood collected from individuals within Sulawesi, Indonesia [11], hereafter referred to as the Yamagishi dataset. Raw sequencing reads for cases $(\mathrm{n}=122)$ and controls $(\mathrm{n}=25)$ were downloaded from SRA studies DRP000987 and DRP001953, respectively.

\section{RNA sequencing data processing}

RNA-seq reads from both studies went through an initial sample quality analysis using FastQC V. 0.11.5 [13]. Leading and trailing bases below a Phred quality score of 20 were removed using Trimmomatic v. 0.36 [14]. RNA-seq reads were then aligned to the human genome (GrCh38, Ensembl release 90: August 2017) with STAR v. 2.5.3a [15] using a two-pass alignment and default parameters. The maximum number of mismatches was set at 10 for the Natri dataset and 3 for the Yamagishi dataset to account for differences in read lengths, which resulted in a mean of 29 million and 17 million uniquely-mapped read pairs for the Natri and Yamagishi datasets, respectively (Supplementary Table 1). All reads which did not map to the human genome were then remapped with STAR [15] to a combined P. falciparum (PF3D7) and P. vivax (PVP01) genome obtained from PlasmoDB (release 36) [16]. The following additional flags were added to account 
for small genome size: genomeSAindexNbases $=11$, alignIntronMax $=35,000$ and alignMatesGapMax $=$ 35,000. We assigned reads to the genome with the highest mapping quality; when reads mapped to both genomes with the same mapping quality, they were assigned to both species.

We performed human read quantification with featureCounts v. 1.5.3 [17] and used a filtered GTF file which only included GENCODE basic (release 27; August 2017) annotation with transcript support levels 1-3. We then used the default settings for single (Yamagishi) and paired end (Natri) reads. On average, we successfully assigned 15 million paired-end reads to each sample from the Natri dataset and 13 million for the Yamagishi dataset (Supplementary Table 1).

For the Natri dataset, library preparation was performed using the Globin-Zero Gold rRNA Removal Kit (Illumina), however a similar depletion step was not performed during the library preparation stage for the Yamagishi dataset. We therefore bioinformatically removed all globin genes from the study (HBA1, $H B A 2, H B B, M B, C Y G B$, and $N G B$ ) which resulted in a more continuous distribution of the total mRNA read pool (Supplementary Figure 1). The minimum library size was then set to 9 million reads post globin removal, which was the minimum number of reads in the Natri dataset. This reduced the total sample size of the Yamagishi dataset from 147 samples to 95.

\section{Deconvolution analysis}

In order to control for heterogeneity within blood cell type proportions and parasite developmental stages, we performed blood and parasite stage deconvolution as follows: For whole blood, we used DeconCell v. 0.1.0 [18] to estimate the proportion of granulocytes, B cells (CD19+), CD4+ T cells, CD8+ T cells, natural killer (NK) cells, and monocytes (range of deconvoluted proportions: $82.5 \%-99.9 \%$ for the Yamagishi data and $89.4 \%-100 \%$ for the Natri data, the latter numbers were taken from [12]). Estimates of the predicted cell proportions are available in Supplementary Table 2. To assign parasite life cycle stage, we used a file from Aunin et al. [19] which contains single cell CPM-normalised gene expression measurements calculated across 12 different parasite stages taken from Plasmodium berghei. Since this study focuses on the human whole blood transcriptome, only Plasmodium stages within the human blood cell stage were used (male and female gametocyte, trophozoite, ring, merozoite, and schizont stages). P. vivax and P. falciparum gene names were converted to one-to-one orthologous $P$. berghei gene names using an orthologous gene file obtained from the Malaria Cell Atlas [20]. We then removed all samples which had no reads mapping to either P. falciparum or P. vivax and uploaded the CPM-normalised counts to CIBERSORT [21] using 100 permutations and disabling quantile normalisation (as recommended on the CIBERSORT page). This was 
performed separately for reads mapping to $P$. vivax and $P$. falciparum, and reads mapping equally well to either species were included in both files. The output from CIBERSORT can be viewed in Supplementary Table 3.

\section{Sex classification}

Due to missing sex information within the Yamagishi dataset, we assigned sex based on expression of marker genes and clustering of reads mapping to the $\mathrm{X}$ and $\mathrm{Y}$ chromosomes. The XIST gene was used as a female expression marker [22] and RPS4Y1, EIF1AY, DDX3Y, and KDM5D were used as male expression markers [23]. This resulted in 8 unlabelled samples mapping to the female group and 5 unlabelled mapped to the male group.

\section{Defining malaria samples and species identification}

The malaria infection status of samples was assessed as follows: in the Natri dataset, four samples showed evidence of Plasmodium infection through nested PCR - a method considered to be the "gold standard" for Plasmodium detection [24]. We then quantified the fraction of reads across these four individuals that mapped to the combined Plasmodium genomes (total number of mapped reads/total number of reads in the starting library), and used this as a threshold for classifying samples as having malaria. All samples which passed this initial threshold $(0.04 \%)$ also had to have reads mapping to a minimum number of genes, where the PCR-confirmed sample with the lowest number of genes $(\mathrm{n}=807)$ was again used as the baseline. Using this criteria, six samples in this dataset were designated as having malaria (For the full reassignment of samples, see Supplementary Table 4).

For the Yamagishi dataset, the authors originally confirmed malaria status through either a rapid malaria paper test or by a smear test. Both of these tests are well documented as commonly having false positives and false negatives [25-27]. Therefore, we classified malaria status by the following: since control samples also had a low number of reads mapping to Plasmodium, we took the control sample with the highest fraction of reads $(0.32 \%)$ and number of genes $(\mathrm{n}=506)$ mapping to Plasmodium. Any sample that did not exceed both of these thresholds was considered to be healthy. Using this criteria, one sample appeared to be healthy and was removed from the analysis, as it could potentially reflect the expression profile of another illness (all sick individuals from the Yamagishi study were collected within a hospital and self-proclaimed sick; For the full reassignment of samples, see Supplementary Table 4.)

Finally, to assess whether individuals were infected with $P$. falciparum or $P$. vivax, we took into account 
the number of reads mapping to either genome for each individual (Supplementary Table 5), as well as clustering by PCA. Samples were first classified as infected by either P. falciparum or P. vivax if over $50 \%$ of Plasmodium reads mapped to that species. PCA using the Plasmodium data, (Supplementary Figure 2) confirmed this assignment, with samples assigned to either species seen to form two distinct clusters, and samples classified as healthy (as above) forming a third. The exception to this was within three samples belonging to the Yamagishi data (DRR006381, DRR006392, and DRR006431). Although these three samples had more reads mapping to $P$. vivax, they were seen to cluster with $P$. falciparum samples in PCA, as well as hierarchical clustering of the Plasmodium reads (Supplementary Figure 2). These samples were therefore classified as having P. falciparum infection.

\section{Differential expression analysis}

We set filtering parameters to only retain genes that were expressed at a CPM $\geq 1$ in at least half of individuals from designated case or control groups. Using these parameters, we kept 12,895 genes from the Yamagishi dataset and 12,743 from the Natri dataset. We then merged both datasets and normalised the library using TMM normalisation [28], resulting in a matrix of 11,735 genes.

To account for variables affecting gene expression levels, we used an ANOVA to test for the association between the first three principal components of the data which captured over $70 \%$ of the variation and all known covariates. Age, which is associated with malaria severity [29], was not available for nine individuals from the Yamagishi study. We therefor removed these nine samples from the dataset, resulting in a total of 201 samples (plus 6 replicates). The breakdown of samples can be viewed in Table 1.

Table 1. Number of samples by sex, study, and location after filtering.

\begin{tabular}{ccccc}
\hline Island & Study & Sex & Case (Control) & Samples \\
\hline Mentawai & Natri & $\mathrm{M}$ & $0(48)$ & 48 \\
\hline Sumba & Natri & $\mathrm{M}$ & $1(48)$ & 49 \\
\hline New Guinea Island & Natri & $\mathrm{M}$ & $5(14)$ & 19 \\
\hline Sulawesi & Yamagishi & $\mathrm{F}$ & $20(10)$ & 30 \\
& Yamagishi & $\mathrm{M}$ & $43(12)$ & 55 \\
\hline Total & & & & $\mathbf{2 0 1}$ \\
\hline
\end{tabular}

After accounting for variables affecting gene expression, the final linear model used to test for differential expression was the following:

Expression $\sim$ species + island + age + sex + falciparum stage proportion (female, male, merozoite, ring, schizont, trophozoite) + vivax stage proportion (female, male, merozoite, ring, schizont, trophozoite) + blood cell type proportion (CD8T, CD4T, NK, B cells, monocytes and granulocytes) 
As this model contains a large number of parameters (22), we wanted to ensure that we were not overfitting it to the data. Therefore, we also tested 3 additional models with reduced parameters using Akaike information criterion (AIC [30]) from the selectModel function in limma v. 3.42 .2 [31]: removing blood deconvolution estimates, removing Plasmodium stage estimates, and removing blood and Plasmodium stage estimates. We found that the full model including all parameters greatly outperformed all other models $(6,803$ genes were associated with the full model being the best fit, followed by 4,341 genes in favour of a model without Plasmodium stage estimates, 324 genes for a model with only species, island, age, and sex information, and 267 genes for a model without blood proportion estimates). For a full breakdown of the estimated proportion of variance each covariate contributed to expression levels, see Supplementary Figure 3.

Differential expression analysis was then conducted using limma with high sample variability removed using the Voom function [32]. We used the technical replicates from the Natri dataset to capture the random individual effect using the limma function duplicateCorrelation [33]. An absolute $\log _{2}(\mathrm{FC})$ threshold of 1 and an FDR-corrected (Benjamini-Hochberg [34]) p-value of 0.05 was used in order to identify differentially expressed genes.

\section{Gene set analysis}

To explore enriched gene sets, we used ClueGo v. 3.8.0 [35], a Cytoscape plugin to decipher GO networks and pathways. The following parameters were used for the GO analysis: Statistical Test Used = Right-sided hypergeometric test for enrichment; Correction Method = Benjamini-Hochberg; Minimum Number of Genes $=4$; Minimum Percentage of Genes $=2 \%$. To analyse biological pathways, we used Reactome, a curated database of biological pathways [36,37].

\section{Comparison of the Indonesian gene sets to other gene sets}

In order to assess whether the Indonesian gene sets were similar to other differential expression analyses, we compared the $P$. falciparum and $P$. vivax gene sets to three other publicly-available studies conducted on uncomplicated malaria patients versus healthy controls. The first study was performed on febrile adults infected with P. falciparum within Mali, an endemic malaria region [38]. Since samples from children are also present in this study, we also collected data from $P$. falciparum-infected children under the age of 10 in Benin [4]. For the P. vivax data, we retrieved data from a controlled human malaria infection (CHMI) study conducted on malaria exposed and malaria naive individuals within Colombia [39]. In order to allow 
bioRxiv preprint doi: https://doi.org/10.1101/2021.01.07.425684; this version posted January 8, 2021. The copyright holder for this preprint

(which was not certified by peer review) is the author/funder, who has granted bioRxiv a license to display the preprint in perpetuity. It is made available under aCC-BY-NC 4.0 International license.

for differences in study design, we did not use an absolute $\log _{2} \mathrm{FC}$ threshold cut-off in comparison to these studies and only filtered for genes with an FDR-corrected p-value less than 0.05. 


\section{Results}

\section{Exploration and control of covariates influencing expression levels}

In order to provide a more comprehensive understanding of malaria pathogenesis in Island Southeast Asia, we have combined data from two RNA-seq studies from malaria-infected and control samples from the Indonesian archipelago. We reanalysed whole blood transcriptomes obtained from 69 Indonesian individuals with simple forms of malaria (asymptomatic and uncomplicated), infected with P. falciparum and P. vivax and compared them to 132 healthy controls. After excluding samples with low library sizes and removing lowly-expressed genes, this resulted in a median library size of $\approx 15$ million for human reads (range: $\approx$ $9 \times 10^{6}-3.2 \times 10^{7}$ ) and $\approx 600,000$ for reads mapping to the $P$. falciparum and $P$. vivax genomes (range: $\approx$ $\left.8 \times 10^{3}-9 \times 10^{6}\right)$.

To explore which variables might be driving variation within the data, we performed principal component analysis (PCA) on the human $\log _{2}$-normalised CPM values. As expected, study had the strongest association with expression levels (Supplementary Figure 4; Supplementary Table 6). Island also had a highly significant association with expression levels, however this was driven predominantly by study, i.e., the island of Sulawesi (Yamagishi) versus the other three islands: Mentawai, Sumba, and the Indonesian half of New Guinea Island (Natri; Supplementary Figure 4). We also observed that leukocyte proportions, which are influenced by infection, were all significantly associated with expression levels (Supplementary Table 6).

As parasite stage can influence gene expression [3,40,41], we deconvoluted the Plasmodium read data through a reference signature gene file from Aunin et al. [19]. We found that for both the P. falciparum and P. vivax data, the most predominant stages were early stage circulating forms of the parasite (merozoites and ring stages, Figure 1, A and B). This aligns with previous research from the Malaria Cell Atlas which has shown that in P. falciparum bulk data, the majority of the cell population is within early stage forms. For P. falciparum, the merozoite stage - the stage in which the parasite is directly exposed to the host immune system [42]—was most prevalent.

We observed a significant difference between control and case samples in levels of B cells, CD4T cells, NK cells, and monocytes $\left(\mathrm{BH}\right.$-corrected ANOVA p $=0.018,5.7 \times 10^{-4}, 0.007$, and $1.4 \times 10^{-4}$, respectively; Figure 1, C). Out of these cell types, control samples had the higher cell type proportion with the exception of monocytes, which were higher in individuals with malaria. Tukey's HSD post-hoc test on CLR-transformed data resulted in significant levels for CD4T cells, CD8T cells, NK cells, and monocytes (BH-corrected ANOVA $\mathrm{p}=0.005,0.018,0.017$, and $5.1 \times 10^{-4}$, respectively), with control samples having higher levels of 
A

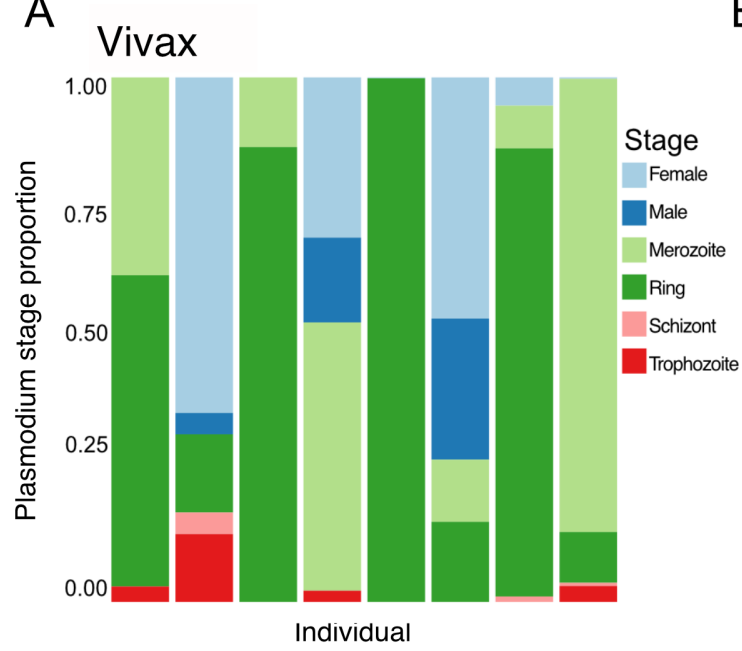

C

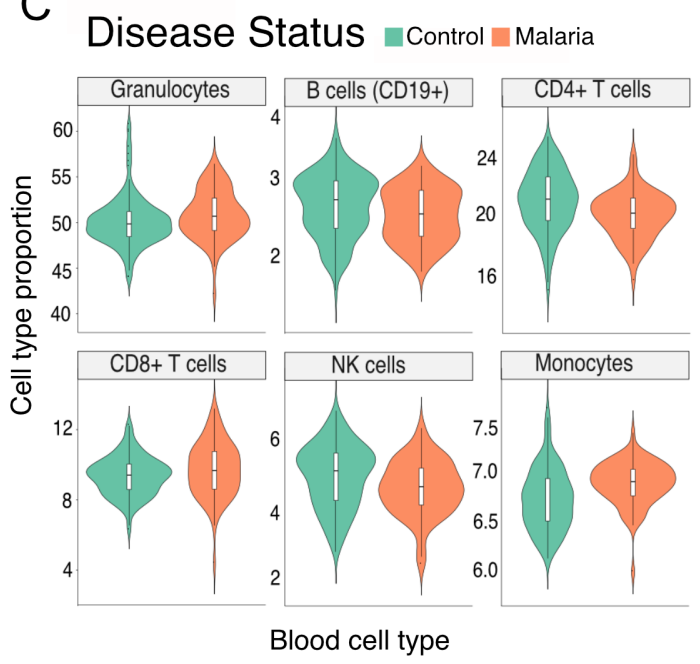

B
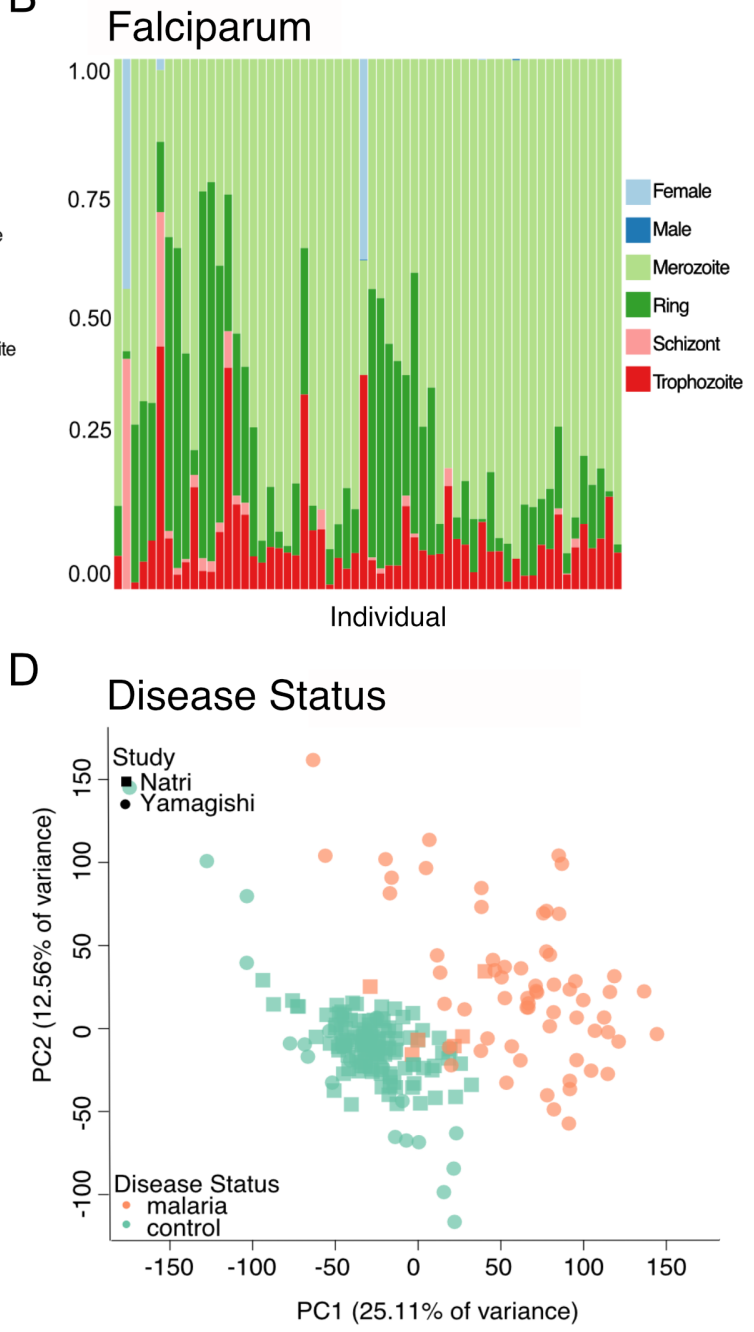

Fig 1. Covariates influencing expression levels. A) Estimated Plasmodium stages within P. vivax and B) P. falciparum. C) Violin plots showing the proportion of blood cell types for each leukocyte within control and malaria-infected individuals. D) PCA analysis of disease status after correcting for batch effects within control (green) and malaria (orange) individuals.

CD4T cells and NK cells, and cases having higher levels of CD8T cells and monocytes (Supplementary Table 2). As noted in previous studies, this increase in monocytes within cases may indicate a high pro-inflammatory immune response [43] and may be protective in order to decrease parasite burden [44, 45].

After adjusting for island, sex, age, stage proportion, and blood cell type proportion, we found that disease status captured most of the remaining variation in the data, followed by Plasmodium species (Supplementary Table 6). Grouping by PCA showed two distinct clusters for control and case samples, with samples from both studies clustering in either group (Figure 1, D). Although we observed clustering between both control and case groups, we observed more variance in the case group than in the control group. We attribute some of this observation to differences in disease severity between malaria-infected individuals; 
Table 2. Number of differentially expressed genes in P. falciparum and P. Vivax-infected individuals compared to healthy controls at an adjusted p-value $(\mathrm{BH})$ of 0.05 and a $\log _{2} \mathrm{FC}$ threshold of 1 (no $\log _{2} \mathrm{FC}$ in parentheses).

\begin{tabular}{ccc}
\hline & Falciparum vs Control & Vivax vs Control \\
\hline Down & $345(1,083)$ & $112(293)$ \\
NotSig & $10,946(9,551)$ & $11,371(10,897)$ \\
Up & $444(1,101)$ & $252(455)$ \\
\hline
\end{tabular}

Yamagishi et al. [11] reported a linear association between malaria severity and Plasmodium-derived read abundance within their samples. Furthermore, as the majority of malaria samples are drawn from the Yamagishi data, which is single-ended and has a shorter read length than the Natri data, the higher variance within the malaria samples could be due to more variation in alignment confidence, particularly across highly variable regions of the genome [46]. This is supported by the fact that the Natri dataset had a much higher proportion of reads that mapped uniquely to the human genome (median $=87 \%$ ) compared to the Yamagishi dataset $($ median $=58 \%$; Supplementary Table 1$)$.

\section{Enriched genes in $P$. falciparum and $P$. vivax are involved in the immune response, translation, and apoptosis}

As we are interested in the biological response to malaria within the Indonesian population, we evaluated the magnitude and significance of differentially expressed genes between cases and controls and separated this into the different infecting Plasmodium species: P. falciparum and P. vivax. Table 2 summarises the results of differential gene expression testing in both group comparisons. At a $\log _{2} \mathrm{FC}$ threshold of 1 , a total of 1,003 genes were differentially expressed in either patients infected with $P$. vivax or patients infected with $P$. falciparum (2,559 genes without any threshold). Of these, 150 genes (373 genes without any threshold) were shared, 639 were unique to P. falciparum (1,811 genes without any threshold), and 214 were unique to $P$. vivax (375 genes without any threshold).

Many of the genes which were DE in both $P$. falciparum and $P$. vivax were part of Reactome pathways related to signalling by interleukins, translation, nonsense mediated decay, and diseases such as influenza (Supplementary Table 7). As identified by Reactome pathway analysis, these shared genes contribute to pathways involved in malaria infection, some of which include regulation of apoptosis [47], inflammation and the immune response [48], and cellular response to environmental stress [49]. For a full list of shared genes, see Supplementary Table 8.

Of all Plasmodium species capable of infecting humans, P. falciparum is responsible for the most severe form of malaria [50], and therefore we investigated the effects of this species versus healthy controls 
A

Falciparum vs Healthy Controls

- NS $\log _{2} \mathrm{FC} \odot \mathrm{p}$-value $\bigcirc \mathrm{p}$-value and $\log _{2} \mathrm{FC}$

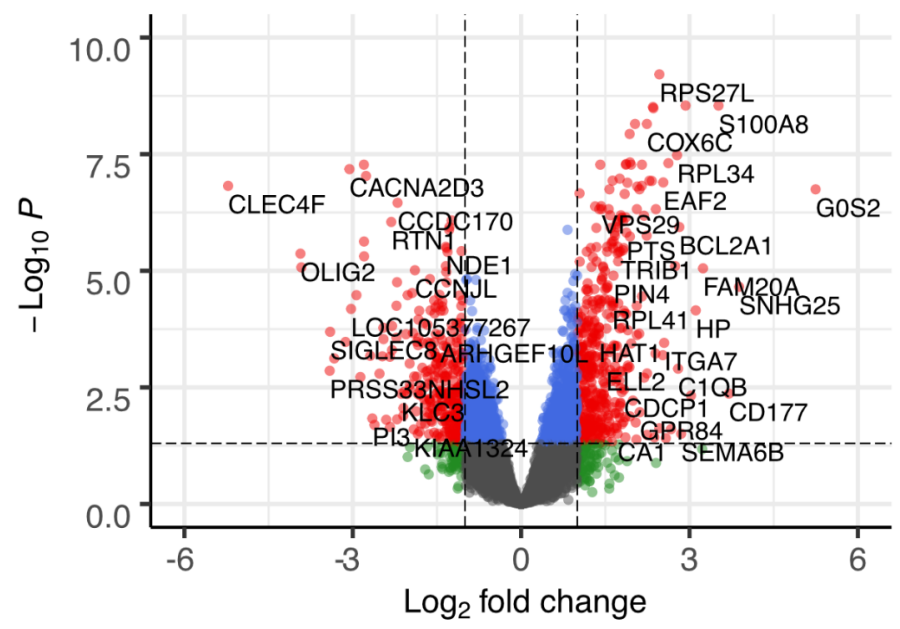

$\mathrm{B}$

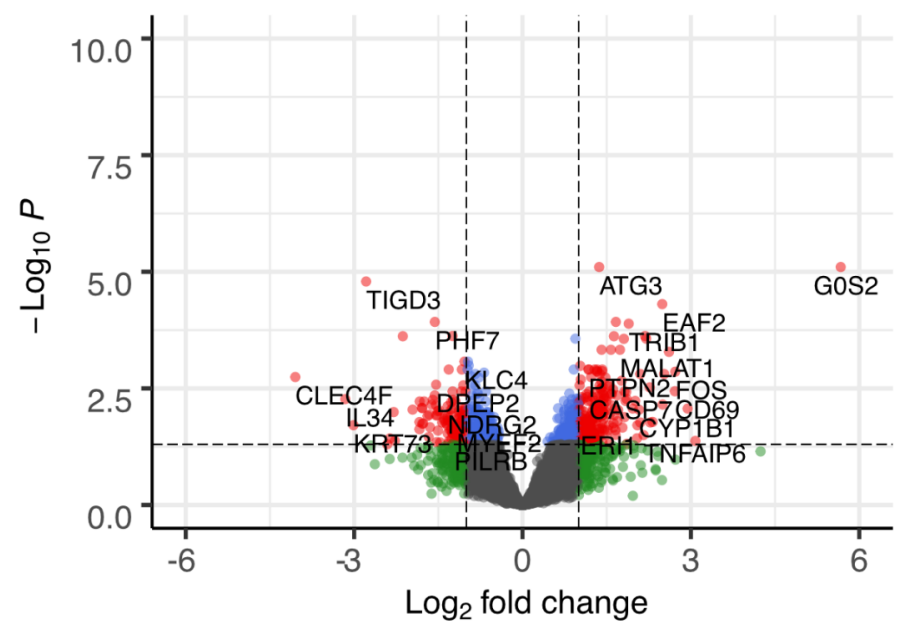

Fig 2. Genes and pathways enriched in P. falciparum and P. vivax-infected individuals. A) Volcano plot of significant genes (indicated in red) at an absolute $\log _{2} \mathrm{FC}$ of 1 and a p-value cut-off of 0.05 for $P$. falciparum and B) P. vivax-infected individuals.

separately. Some of the most highly upregulated genes in P. falciparum-infected individuals included immediate early response genes such as FOS [5] and DUSP1 [51], as well as genes involved in apoptosis (some of the most highly upregulated genes being G0S2 and BCL2A1). We also observed a strong signal from inflammation-related genes; SOCS3 and IFNG, two major regulators of infection and inflammation $[52,53]$, were significantly upregulated in P. falciparum patients. Furthermore, inflammatory chemokines, which are well-documented to have an association with malaria [54], were both significantly up and down regulated (Figure 2). For example, expression of CXCL8 was upregulated, as noted in other studies [54-56], while CCR3 and CCR4 were downregulated, an effect which has previously been observed [38].

Some of the most highly upregulated genes in our data were genes encoding ribosomal proteins, as well as genes involved in translation pathways (Supplementary Table 9). In addition, some of the most significantly-enriched Reactome pathways in P. falciparum patients were those involved in translation and nonsense mediated decay (Supplementary Table 7), and GO analyses were enriched in the regulation of apoptotic processes and translation (Supplementary Table 10). Since ribosomal protein genes are from large gene families [57], we tested whether this signal could be driven by differences between cases and controls in read length (the majority of reads from cases being 36 bp long, while those from controls being 100 bp long in most cases). We found that when we stratified cases and controls by read length, mean expression of significant DE genes involved in translation (beginning with RPL, MRPL or RPS; $\mathrm{n}=85$ ) amongst sample 
classes were still significantly different (Tukey's HSD on 36 bp cases versus 100 bp controls ANOVA p = $3.4 \times 10^{-8}$; Supplementary Figure 6), suggesting a biological response rather than sequencing artefact.

Due to differences in biology to P. falciparum $[58,59]$, we also analysed the effects of infection with P. vivax compared to healthy controls. We found that Reactome pathways were significantly enriched in pathways related to interleukin signalling (Supplementary Table 7), while GO terms were enriched in cellular response to stress, regulation of metabolic processes, response to cytokines, translation, and apoptotic processes (Supplementary Table 10).

As above, some of the most highly enriched genes in $P$. vivax patients were those involved in the immediate early immune response, inflammatory response, and translation (Supplementary Table 9). For all shared genes, we also found changes due to $P$. vivax infection to have the same direction of effect and similar $\log _{2}$ FC intensities to those observed in P. falciparum patients (Supplementary Figure 5). However, some differences with the $P$. falciparum response were also apparent: We found that $P$. falciparum-infected individuals had a much stronger signal in the defense and inflammatory response (Supplementary Table 9, Supplementary Table 10), as shown by an upregulation of chemokines and IFNG, both of which were absent in P. vivax-infected individuals. ICAM1 and the complement component genes $C 1 Q A$ and $C 1 Q B$, all of which have previously been associated with malaria severity [60,61], were also upregulated exclusively in $P$. falciparum-infected patients. Finally, we found that CD69, a marker of recent T-cell activation which has previously been associated with less severe forms of malaria [62], was one of the most highly upregulated genes in $P$. vivax-infected individuals $\left(\mathrm{BH}\right.$-adjusted $\mathrm{p}=1.9 \times 10^{-5}$ ), but not significantly differentially expressed in P. falciparum-infected patients (BH-adjusted $\mathrm{p}=0.052)$.

\section{Comparing the Indonesian response to malaria infection to other populations}

In order to identify any possible Indonesian-specific component of this response, we compared genes differentially expressed in Indonesia in response to $P$. falciparum and $P$. vivax infection to four other publiclyavailable datasets of differentially expressed genes, taken from three studies conducted on uncomplicated malaria patients versus healthy controls $[4,38,39]$. The first two datasets are from P. falciparum-infected individuals who have either previously had malaria exposure (malaria-exposed individuals), collected from individuals in Mali [38], or individuals who were never exposed to malaria (malaria-naive), taken from children in Benin [4]. The other two datasets are from P. vivax-infected individuals from Colombia, collected from both individuals living in malaria-endemic regions in Buenaventura (malaria-exposed), as well as individuals from Cali with no previous malaria exposure (malaria-naive) [39]. In all cases, the available 
A

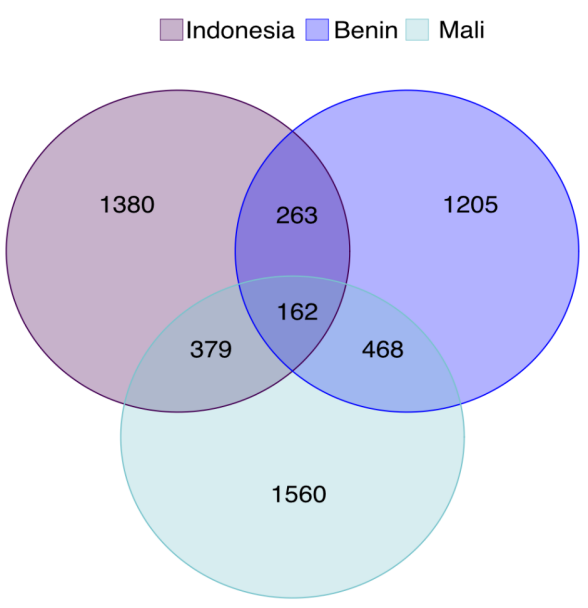

6318

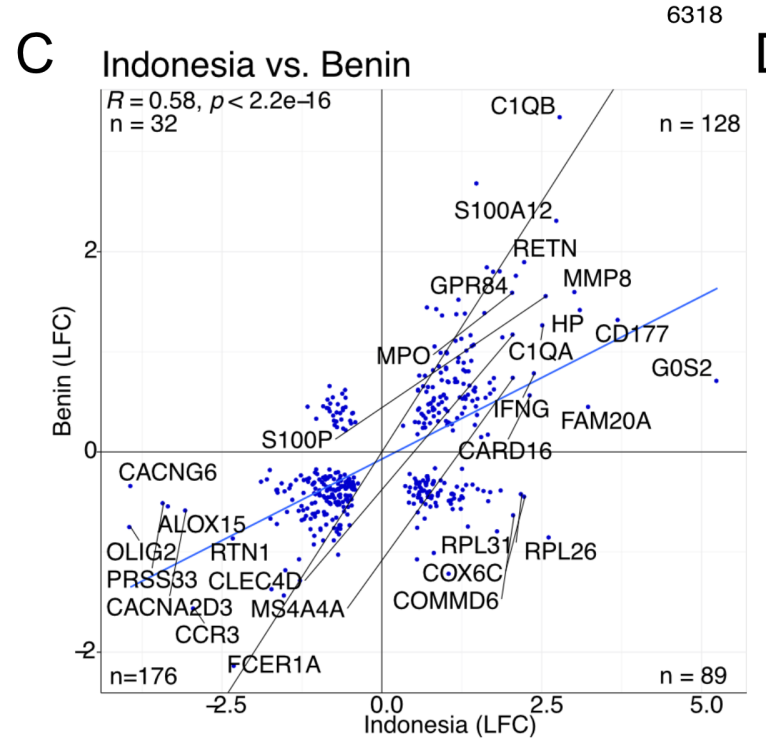

E Indonesia vs. Mali

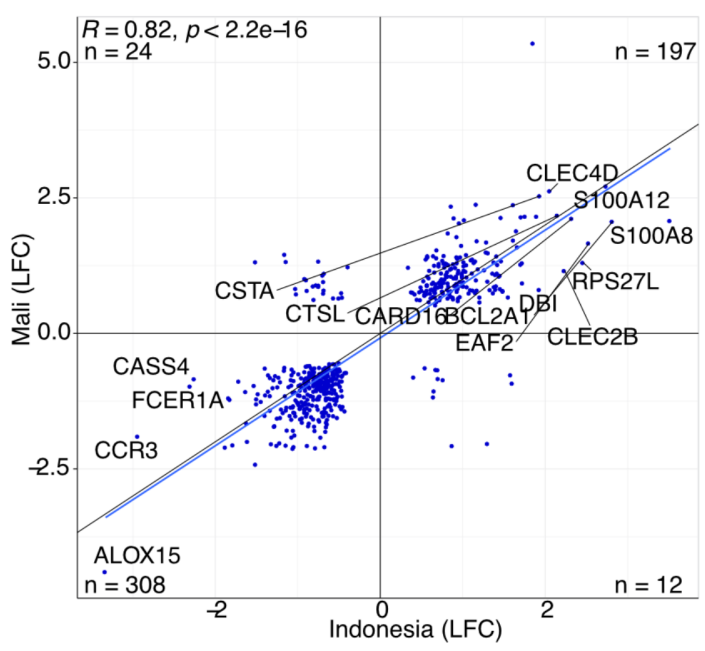

B

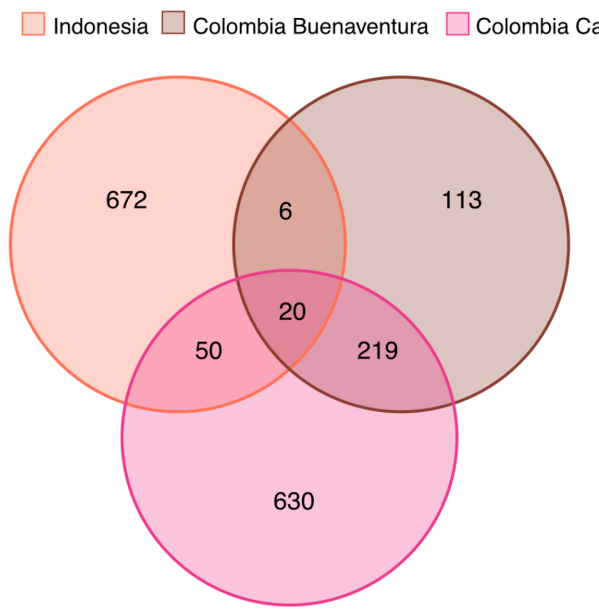

10025

Indonesia vs. Colombia (Buenaventura)

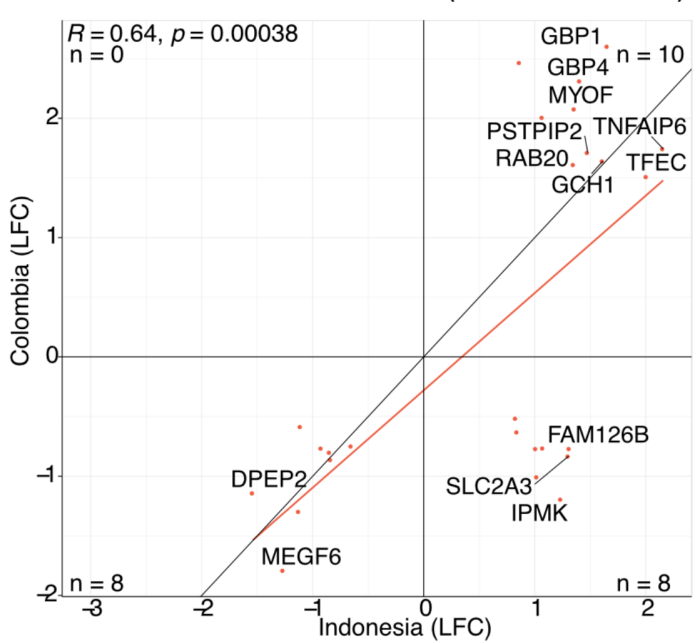

F Indonesia vs. Colombia (Cali)

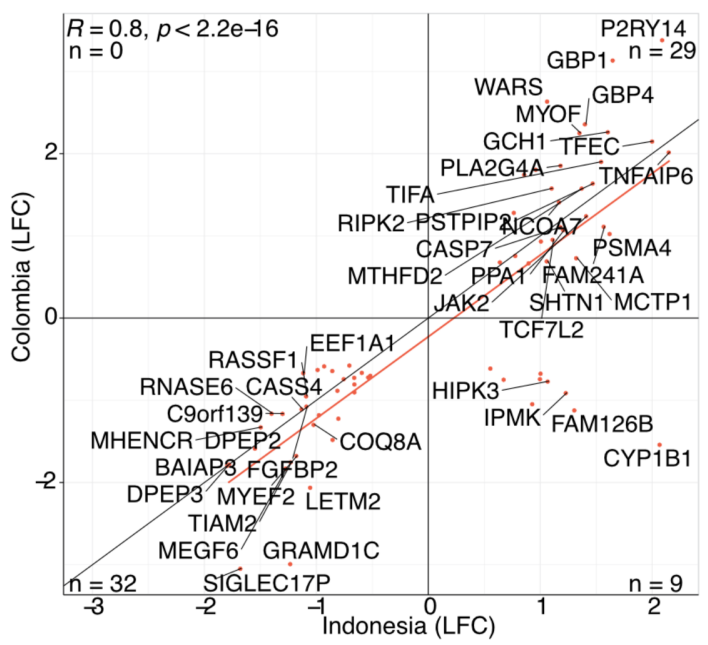

Fig 3. Comparisons between Plasmodium-infected patients in Indonesia and other malaria-endemic countries. A) Total number of differentially expressed genes between P. falciparum-infected individuals and healthy controls in Indonesia, Benin, and Mali (FDR $\leq 0.05$ and no absolute $\log _{2}$ FC). B) Total number of differentially expressed genes between $P$. vivax-infected individuals and healthy controls in Indonesia, Cali, Colombia, and Buenaventura, Colombia (FDR $\leq 0.05$ and no absolute $\log _{2}$ FC). C-F) Comparison of enriched genes (FDR $\leq 0.05$ and no absolute $\log _{2} \mathrm{FC}$ ) within Indonesian populations and populations outside of Island Southeast Asia [4,38,39]. Panels C and E are enriched genes for P. falciparum while panels $\mathrm{D}$ and $\mathrm{F}$ are for genes enriched in $P$. vivax. 
data is the list of differentially expressed genes between patients and healthy controls, and we tested for overlap between lists without thresholding on $\log _{2} \mathrm{FC}$ values.

For comparisons to $P$. falciparum-exposed individuals from Mali, we began with a list of 2,775 genes found to be differentially expressed by the authors $(\mathrm{p}=0.05$; number of individuals $=3$; RNA-seq measurements). Of these, 541 genes were differentially expressed in our own data out of 2,569 genes $(21.06 \%)$ which were present in both datasets. For comparisons to P. falciparum-exposed individuals from Benin, we took a list of 2,351 genes found to be differentially expressed by the authors $(\mathrm{p}=0.05$; number of individuals $=$ 94; microarray measurements). Of these, 425 genes were differentially expressed in our own data out of 2,098 genes (20.26\%) which were present in both datasets (Figure 3). Many of these genes are involved in apoptosis, inflammation, and the innate immune system. Furthermore, we found many of these genes shared the same direction of effect and had a similar magnitude of expression. This is particularly true in comparison to the population within Mali, where nearly all genes shared the same direction of effect and had similar trends in fold change $(\mathrm{R}=0.82)$.

For comparisons to $P$. vivax-exposed individuals from Colombia, Cali who are malaria-naive, we took a list of 1,072 genes found to be differentially expressed by the authors ( $\mathrm{p}=0.05$; number of individuals $=6$; RNA-seq measurements). Of these, 70 genes were differentially expressed in our own data out of 919 genes $(7.62 \%)$ which were present in both datasets. For comparisons to $P$. vivax-exposed individuals from Colombia, Buenaventura who had prior malaria exposure, we took a list of 400 genes found to be differentially expressed by the authors ( $\mathrm{p}=0.05$; number of individuals $=6$; RNA-seq measurements). Of these, 26 genes were differentially expressed in our own data out of 358 genes (7.26\%) which were present in both datasets (Figure 3). Many of these genes are involved in innate immunity, such as the interferon-induced genes GBP1 and GBP4, as well as JAK2. Other shared genes include apoptosis-related genes and transport genes (Figure 3). We found many more genes were shared between populations from Cali, Colombia (the malaria-naive population) than populations from Buenaventura, Colombia (the malaria-exposed population), as well as a stronger correlation in $\log _{2}$ FC magnitude and direction $(\mathrm{R}=$ $0.8)$.

In our comparison of responses to infection with $P$. vivax and $P$. falciparum, we found that many differentially expressed genes were shared across populations in response to infection by either species. In order to test, on a broader scale, which genes are shared between not only species but also different global regions, we compared all significant genes (FDR $\leq 0.05$ with no $\log _{2}$ FC threshold) within the Indonesian population to the African (combining the differentially expressed genes within Benin and Mali for a total 
bioRxiv preprint doi: https://doi.org/10.1101/2021.01.07.425684; this version posted January 8, 2021. The copyright holder for this preprint (which was not certified by peer review) is the author/funder, who has granted bioRxiv a license to display the preprint in perpetuity. It is made available under aCC-BY-NC 4.0 International license.
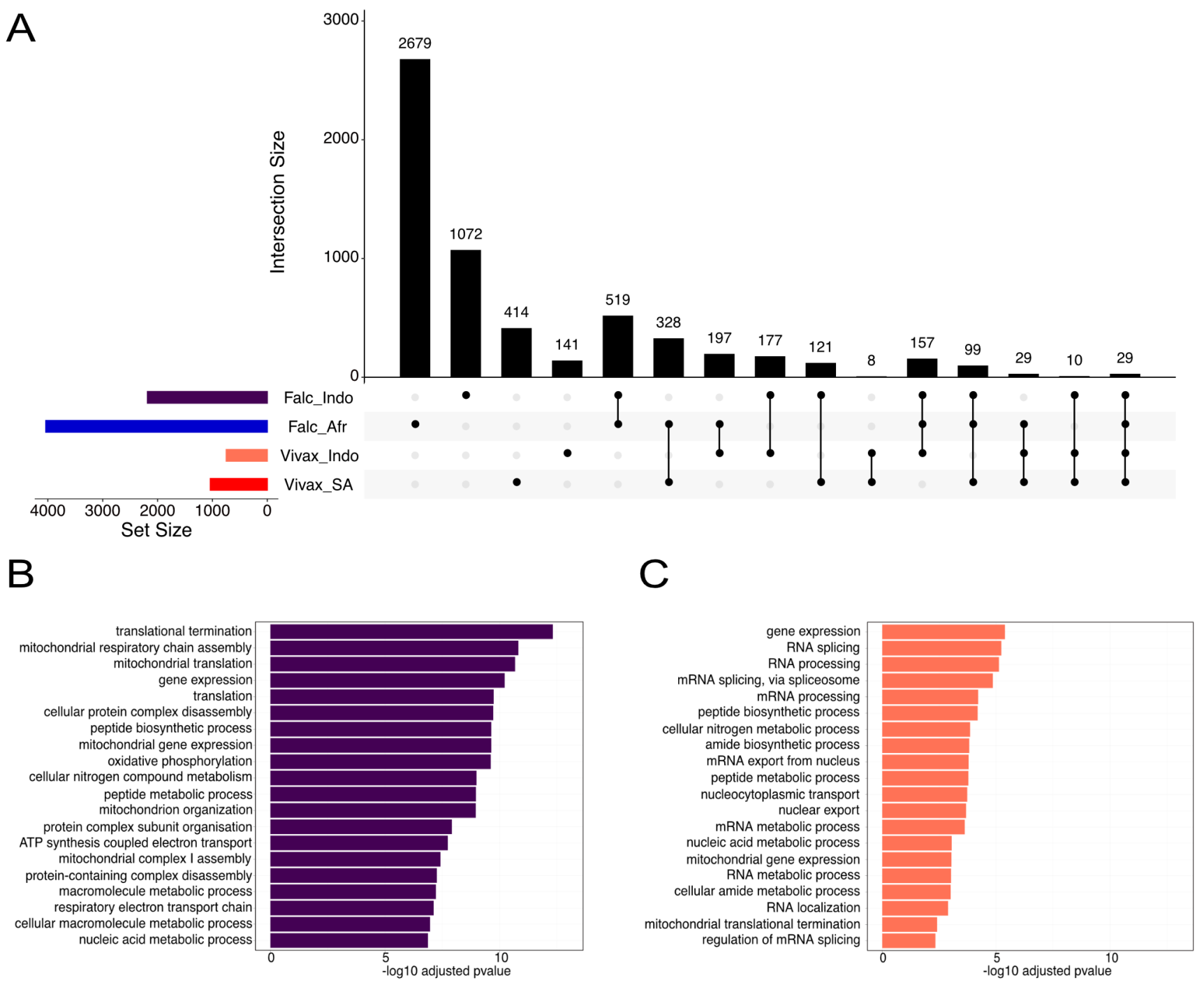

Fig 4. Shared and Indonesian-specific genes within P. vivax and P. falciparum-infected patients. A) Shared and unique genes across multiple populations. P. falciparum and P. Vivax-infected individuals within Indonesia compared to $P$. falciparum and $P$. vivax-infected individuals within South America (Colombia) and Africa (Mali and Benin). The number of shared genes for each comparison can be viewed on the top of each bar. B) Gene ontology enrichment terms (BP) for unique genes found within Indonesian individuals infected with $P$. falciparum. The top 20 terms by adjusted p-value (BH) are shown. C) Gene ontology enrichment terms (BP) for unique genes found within Indonesian individuals infected with $P$. vivax. The top 20 terms by adjusted p-value $(\mathrm{BH})$ are shown.

list of 4,037 genes) and South American populations (composed of the differentially expressed genes within the Colombian populations of Cali and Buenaventura, again combined, 1,038 genes). We found that many genes are unique to the Indonesian populations (unique to $P$. falciparum infection versus all non-Indonesian datasets $=1,072$; unique to $P$. vivax infection versus all non-Indonesian datasets $=141 ;$ common across $P$. vivax and $P$. falciparum infection but absent from all non-Indonesian datasets $=177$ ), although we observed a substantial overlap across the populations in our study (Figure 4). Indonesian populations infected with 
either P. falciparum or P. vivax had the highest similarity with populations from Africa. Furthermore, 29 genes were shared between all species and all populations, suggesting that despite Plasmodium species and genetic background, some host genes are integral during Plasmodium infection (see Supplementary Table 8).

Finally, we wanted to investigate those genes whose upregulation appears unique to malaria-infected individuals within Indonesia, $(\mathrm{n}=1,213$; Figure 4). Although differences in study design and technology between studies affects our ability to definitively attribute these genes as being Indonesian-specific, exploring these genes allows for an initial investigation of potential population-specific pathways. For individuals infected with P. falciparum, we found many of these genes were enriched in GO pathways related to RNA processing and splicing (Figure 4; for a full list of enriched GO terms, see Supplementary Table 10), as well as Reactome pathways involved in mitochondrial translation, respiratory electron transport, and activation of ATR in response to replication stress (Supplementary Table 7). For genes specific to P. vivax, we found the majority of genes were also enriched in GO terms and Reactome pathways involved in RNA processing and splicing, as well as translation and protein transport (Figure 4; Supplementary Table 7; Supplementary Table 10). Indeed, genes such as Sm-like protein genes and SR protein genes, which are crucial to RNA splicing, were upregulated within $P$. falciparum and $P$. vivax-infected patients. Other notable genes include surface expressed protein genes such as $C L E C 4 F$ and $S I G L E C 8$, which had some of the $\operatorname{largest}_{\log _{2}}$ FC values. (The full list of genes unique to individuals infected with P. falciparum and P. vivax can be found in Supplementary Table 9.) 


\section{Discussion}

Our understanding of the human immune response to malaria infection remains in its infancy. Here, we have investigated the transcriptional response to simple forms of malaria within a large group of Indonesian individuals and find both conserved and unique components to it, relative to other human groups. We found a total of 789 genes differentially expressed in response to infection by P. falciparum and 364 in response to $P$. Vivax, within the range of previous findings.

The innate immune system plays an important role against pathogens by eliciting physical and chemical barriers to protect the host [63], and we found that this was one of the strongest signals observed in our data. Inflammation, a pathway which mediates parasite clearance [64], was evident by the upregulation of IRAK3, S100A8, and SOCS3, all known inflammatory markers and mediators [52,65,66]. This finding corroborates earlier research which has shown that the inflammatory response can be triggered by intracellular parasites which destroy infected cells [48]. Other innate immune signatures included the upregulation of FOS, a gene implicated in the immediate early immune response and immune modulation [67], as well as the upregulation of genes involved in the complement system, a pathway which plays an important role in the inhibition of merozoite invasion [68].

Beyond innate immune responses, we also observed expression of genes involved in apoptosis. Apoptosis is a mechanism which removes damaged cells from the body [69] and is an observed effect during malaria infection [70-72]. Although the role of apoptosis in malaria infection has not been fully elucidated [73], some studies have proposed that apoptosis occurs in response to haemozoin, a waste product of haemoglobin digestion by Plasmodium [71,72,74,75]. Indeed, the upregulation of apoptosis-related pathways and genes, particularly G0S2 which had the highest $\log _{2} \mathrm{FC}$ value in both P. vivax and P. falciparum, could therefore be a response to haemozoin and oxidative stress.

In order to explore similarities in the regulatory mechanisms of malaria infection, we compared differentially expressed genes from P. falciparum and P. vivax-infected Indonesian individuals to three other transcriptomic studies conducted on individuals with uncomplicated forms of malaria and healthy controls from Africa [4,38] and South America [39], bearing in mind the fact that there are substantial differences in sample size, power and study design between all the data sets we considered. We found that many shared genes in P. falciparum-infected individuals were involved in immune-related processes such as receptor recognition genes, chemokine receptors, and inflammation-related genes. In comparisons between P. vivax-infected populations, innate immune genes such as JAK2 and interferon-induced genes, were shared. Malaria is a highly inflammatory disease, and therefore these shared genes may reflect a general 
inflammatory response.

These shared genes exhibited similar direction of effects across studies (Figure 3), which was particularly apparent for malaria-exposed adults from Mali $(\mathrm{R}=0.82)$. Comparisons to the Benin population had a lower correlation coefficient $(\mathrm{R}=0.58)$ to that of Mali, which may be a reflection of technological differences, as the study was conducted using microarray data in 2012 [4]. We also note that our P. vivax patient dataset and the Colombian populations we used for comparison to our $P$. vivax data have small sample sizes ( $\mathrm{n}=6$ and $\mathrm{n}=8$, respectively), which may explain the lower correlations we observed, especially against malaria-exposed individuals from Buenaventura $(\mathrm{R}=0.64$; Figure 3$)$.

We also attempted to identify malaria-associated pathways which are unique to Indonesia. We found that many of the enriched genes unique to the Indonesian population were heavily involved in RNA processing and splicing, and that these differences were not due to differences in sequencing length between groups (Supplementary Figure 6). RNA splicing and its impact on immunity has been described previously (for an extensive review, see [76]) and studies have shown that variation in alternative splicing is common across populations from different ethnic backgrounds [77]. No genes known to be implicated in malaria resistance or susceptibility were found within the Indonesian dataset, however we did find that surface expressed protein genes such as $C L E C 4 F$ and $S I G L E C 8$, which had some of the largest $\log _{2}$ FC values, were unique to the Indonesian dataset. Receptors expressed on immune cells are important mediators of the immune response [78,79], and previous research has implicated other C-type lectins, such as CLEC12A [80] and $C L E C 4 A$ [81], in modulating malaria pathogenesis. This may suggest that the variability in ways that populations differ from one another are still not fully understood and may include variation in genes such as alternative splicing, receptor processing, and binding.

All of the studies we have considered used different approaches to data generation and processing. Despite our best efforts, some unwanted technical and biological variation is likely to remain in the data. However, despite these differences, many of the genes differentially expressed in our Indonesian data after batch correction were also genes with some of the strongest signals in other studies that we compared to. These overlapping genes are involved in antigen clearance, cellular signalling, and inflammation, which are all well-documented pathways involved in malaria infection [82-84]. This may suggest that many crucial immunological pathways during infection are shared across populations, a finding which could be particularly useful for biomarker discovery and diagnostics in the field. While some of the differences we observe between populations may be artefactual, population-specific responses to malaria are well documented and not unexpected [2]. 
Studying the biological response to infection within the human host across a range of genetic backgrounds is crucial for a more comprehensive understanding of disease, and by extension, the efficacy of vaccines [85], drugs [86], and biomarkers [87]. This is particularly true for malaria, where it has been shown that populations vary in their metabolism of drugs and can suffer from severe reactions to antimalarials [88]. Broadening the repertoire of genes known to be implicated in malaria across populations is essential if we aim to better diagnose and treat this disease. By characterising that response in populations within Indonesia, and situating it within the global context, we have shown that the immune response to malaria infection can have shared and population-specific effects, and that considering these population differences is an essential step in creating more broadly effective malaria therapeutics.

\section{Competing interests}

The authors declare that they have no competing interests.

\section{Author's contributions}

KSB and IGR designed the study. KSB performed analyses and wrote the manuscript with input from all authors. DS performed PCR malaria diagnosis. HS and CCD generated the Indonesian datasets.

\section{Acknowledgements}

We would like to acknowledge all of the study participants who generously consented to genome sequencing in the original study. We would also like to thank Pradiptajati Kusuma (Eijkman Institute) for his valuable comments. KSB was supported by a Melbourne Graduate Research Scholarship.

\section{References}

1. Organization, W.H., et al.: World malaria report 2017. 2017. Reference Source (2017)

2. Kwiatkowski, D.P.: How malaria has affected the human genome and what human genetics can teach us about malaria. The American Journal of Human Genetics 77(2), 171-192 (2005)

3. Lee, H.J., Georgiadou, A., Walther, M., Nwakanma, D., Stewart, L.B., Levin, M., Otto, T.D., Conway, D.J., Coin, L.J., Cunnington, A.J.: Integrated pathogen load and dual transcriptome analysis of 
systemic host-pathogen interactions in severe malaria. Science translational medicine 10(447), 3619 $(2018)$

4. Idaghdour, Y., Quinlan, J., Goulet, J.-P., Berghout, J., Gbeha, E., Bruat, V., De Malliard, T., Grenier, J.-C., Gomez, S., Gros, P., et al.: Evidence for additive and interaction effects of host genotype and infection in malaria. Proceedings of the National Academy of Sciences 109(42), 16786-16793 (2012)

5. Boldt, A.B., Van Tong, H., Grobusch, M.P., Kalmbach, Y., Ella, A.D., Kombila, M., Meyer, C.G., Kun, J.F., Kremsner, P.G., Velavan, T.P.: The blood transcriptome of childhood malaria. EBioMedicine 40, 614-625 (2019)

6. Paquette, A., Harahap, A., Laosombat, V., Patnode, J., Satyagraha, A., Sudoyo, H., Thompson, M., Yusoff, N., Wilder, J.A.: The evolutionary origins of southeast asian ovalocytosis. Infection, Genetics and Evolution 34, 153-159 (2015)

7. Hill, A.V., Allsopp, C.E., Kwiatkowski, D., Anstey, N.M., Twumasi, P., Rowe, P.A., Bennett, S., Brewster, D., McMichael, A.J., Greenwood, B.M.: Common west african hla antigens are associated with protection from severe malaria. Nature 352(6336), 595 (1991)

8. Ackerman, H., Usen, S., Jallow, M., Sisay-Joof, F., Pinder, M., Kwiatkowski, D.: A comparison of case-control and family-based association methods: the example of sickle-cell and malaria. Annals of human genetics 69(5), 559-565 (2005)

9. Organization, W.H., et al.: World malaria report 2018. 2018. World Health Organization: Geneva

10. Hudjashov, G., Karafet, T.M., Lawson, D.J., Downey, S., Savina, O., Sudoyo, H., Lansing, J.S., Hammer, M.F., Cox, M.P.: Complex patterns of admixture across the indonesian archipelago. Molecular biology and evolution 34(10), 2439-2452 (2017)

11. Yamagishi, J., Natori, A., Tolba, M.E., Mongan, A.E., Sugimoto, C., Katayama, T., Kawashima, S., Makalowski, W., Maeda, R., Eshita, Y., et al.: Interactive transcriptome analysis of malaria patients and infecting plasmodium falciparum. Genome research 24(9), 1433-1444 (2014)

12. Natri, H.M., Bobowik, K.S., Kusuma, P., Crenna Darusallam, C., Jacobs, G.S., Hudjashov, G., Lansing, J.S., Sudoyo, H., Banovich, N.E., Cox, M.P., et al.: Genome-wide dna methylation and gene expression patterns reflect genetic ancestry and environmental differences across the indonesian archipelago. PLoS Genetics 16(5), 1008749 (2020) 
13. Andrews, S., Krueger, F., Segonds-Pichon, A., Biggins, L., Krueger, C., Wingett, S.: FastQC. Babraham Institute, Babraham, UK (2012)

14. Bolger, A.M., Lohse, M., Usadel, B.: Trimmomatic: a flexible trimmer for illumina sequence data. Bioinformatics 30(15), 2114-2120 (2014)

15. Dobin, A., Davis, C.A., Schlesinger, F., Drenkow, J., Zaleski, C., Jha, S., Batut, P., Chaisson, M., Gingeras, T.R.: Star: ultrafast universal rna-seq aligner. Bioinformatics 29(1), 15-21 (2013)

16. Aurrecoechea, C., Brestelli, J., Brunk, B.P., Dommer, J., Fischer, S., Gajria, B., Gao, X., Gingle, A., Grant, G., Harb, O.S., et al.: Plasmodb: a functional genomic database for malaria parasites. Nucleic acids research 37(suppl_1), 539-543 (2008)

17. Liao, Y., Smyth, G.K., Shi, W.: featurecounts: an efficient general purpose program for assigning sequence reads to genomic features. Bioinformatics 30(7), 923-930 (2013)

18. Aguirre-Gamboa, R., de Klein, N., di Tommaso, J., Claringbould, A., Vosa, U., Zorro, M., Chu, X., Bakker, O.O., Borek, Z., Ricano-Ponce, I., et al.: Deconvolution of bulk blood eqtl effects into immune cell subpopulations. bioRxiv, 548669 (2019)

19. Aunin, E., Böhme, U., Sanderson, T., Simons, N.D., Goldberg, T.L., Ting, N., Chapman, C.A., Newbold, C.I., Berriman, M., Reid, A.J.: Genomic and transcriptomic evidence for descent from plasmodium and loss of blood schizogony in hepatocystis parasites from naturally infected red colobus monkeys. PLoS pathogens 16(8), 1008717 (2020)

20. Howick, V.M., Russell, A., Andrews, T., Heaton, H., Reid, A.J., Natarajan, K.N., Butungi, H., Metcalf, T., Verzier, L.H., Rayner, J., et al.: The malaria cell atlas: a comprehensive reference of single parasite transcriptomes across the complete plasmodium life cycle. BioRxiv, 527556 (2019)

21. Newman, A.M., Liu, C.L., Green, M.R., Gentles, A.J., Feng, W., Xu, Y., Hoang, C.D., Diehn, M., Alizadeh, A.A.: Robust enumeration of cell subsets from tissue expression profiles. Nature methods 12(5), $453(2015)$

22. Ji, B., Higa, K.K., Kelsoe, J.R., Zhou, X.: Over-expression of xist, the master gene for x chromosome inactivation, in females with major affective disorders. EBioMedicine 2(8), 909-918 (2015) 
23. Staedtler, F., Hartmann, N., Letzkus, M., Bongiovanni, S., Scherer, A., Marc, P., Johnson, K.J., Schumacher, M.M.: Robust and tissue-independent gender-specific transcript biomarkers. Biomarkers 18(5), 436-445 (2013)

24. Tangpukdee, N., Duangdee, C., Wilairatana, P., Krudsood, S.: Malaria diagnosis: a brief review. The Korean journal of parasitology 47(2), 93 (2009)

25. Koita, O.A., Doumbo, O.K., Ouattara, A., Tall, L.K., Konaré, A., Diakité, M., Diallo, M., Sagara, I., Masinde, G.L., Doumbo, S.N., et al.: False-negative rapid diagnostic tests for malaria and deletion of the histidine-rich repeat region of the hrp2 gene. The American journal of tropical medicine and hygiene 86(2), 194-198 (2012)

26. Kozycki, C.T., Umulisa, N., Rulisa, S., Mwikarago, E.I., Musabyimana, J.P., Habimana, J.P., Karema, C., Krogstad, D.J.: False-negative malaria rapid diagnostic tests in rwanda: impact of plasmodium falciparum isolates lacking hrp2 and declining malaria transmission. Malaria journal 16(1), 123 (2017)

27. Meatherall, B., Preston, K., Pillai, D.R.: False positive malaria rapid diagnostic test in returning traveler with typhoid fever. BMC infectious diseases 14(1), 377 (2014)

28. Robinson, M.D., Oshlack, A.: A scaling normalization method for differential expression analysis of rna-seq data. Genome biology 11(3), 25 (2010)

29. White, M., Watson, J.: Malaria: Age, exposure and immunity. Elife 7, 40150 (2018)

30. Akaike, H.: Information theory and an extension of the maximum likelihood principle. In: Selected Papers of Hirotugu Akaike, pp. 199-213. Springer, ??? (1998)

31. Ritchie, M.E., Phipson, B., Wu, D., Hu, Y., Law, C.W., Shi, W., Smyth, G.K.: limma powers differential expression analyses for rna-sequencing and microarray studies. Nucleic acids research 43(7), 47-47 (2015)

32. Law, C.W., Chen, Y., Shi, W., Smyth, G.K.: voom: Precision weights unlock linear model analysis tools for rna-seq read counts. Genome biology 15(2), 29 (2014)

33. Smyth, G.K., Michaud, J., Scott, H.S.: Use of within-array replicate spots for assessing differential expression in microarray experiments. Bioinformatics 21(9), 2067-2075 (2005) 
34. Benjamini, Y., Hochberg, Y.: Controlling the false discovery rate: a practical and powerful approach to multiple testing. Journal of the Royal statistical society: series B (Methodological) 57(1), 289-300 (1995)

35. Bindea, G., Mlecnik, B., Hackl, H., Charoentong, P., Tosolini, M., Kirilovsky, A., Fridman, W.-H., Pagès, F., Trajanoski, Z., Galon, J.: Cluego: a cytoscape plug-in to decipher functionally grouped gene ontology and pathway annotation networks. Bioinformatics 25(8), 1091-1093 (2009)

36. Fabregat, A., Jupe, S., Matthews, L., Sidiropoulos, K., Gillespie, M., Garapati, P., Haw, R., Jassal, B., Korninger, F., May, B., et al.: The reactome pathway knowledgebase. Nucleic acids research 46(D1), 649-655 (2018)

37. Jassal, B., Matthews, L., Viteri, G., Gong, C., Lorente, P., Fabregat, A., Sidiropoulos, K., Cook, J., Gillespie, M., Haw, R., et al.: The reactome pathway knowledgebase. Nucleic acids research 48(D1), 498-503 (2020)

38. Tran, T.M., Jones, M.B., Ongoiba, A., Bijker, E.M., Schats, R., Venepally, P., Skinner, J., Doumbo, S., Quinten, E., Visser, L.G., et al.: Transcriptomic evidence for modulation of host inflammatory responses during febrile plasmodium falciparum malaria. Scientific reports 6, 31291 (2016)

39. Vallejo, A.F., Read, R.C., Arevalo-Herrera, M., Herrera, S., Elliott, T., Polak, M.E.: Malaria systems immunology: Plasmodium vivax induces tolerance during primary infection through dysregulation of neutrophils and dendritic cells. Journal of Infection 77(5), 440-447 (2018)

40. Lemieux, J.E., Gomez-Escobar, N., Feller, A., Carret, C., Amambua-Ngwa, A., Pinches, R., Day, F., Kyes, S.A., Conway, D.J., Holmes, C.C., et al.: Statistical estimation of cell-cycle progression and lineage commitment in plasmodium falciparum reveals a homogeneous pattern of transcription in ex vivo culture. Proceedings of the National Academy of Sciences 106(18), 7559-7564 (2009)

41. Kim, A., Popovici, J., Menard, D., Serre, D.: Plasmodium vivax transcriptomes reveal stagespecific chloroquine response and differential regulation of male and female gametocytes. Nature communications 10(1), 371 (2019)

42. Cowman, A.F., Berry, D., Baum, J.: The cellular and molecular basis for malaria parasite invasion of the human red blood cell. Journal of cell Biology 198(6), 961-971 (2012) 
43. Antwi-Baffour, S., Kyeremeh, R., Buabeng, D., Adjei, J.K., Aryeh, C., Kpentey, G., Seidu, M.A.: Correlation of malaria parasitaemia with peripheral blood monocyte to lymphocyte ratio as indicator of susceptibility to severe malaria in ghanaian children. Malaria journal 17(1), 1-9 (2018)

44. Royo, J., Rahabi, M., Kamaliddin, C., Ezinmegnon, S., Olagnier, D., Authier, H., Massougbodji, A., Alao, J., Ladipo, Y., Deloron, P., et al.: Changes in monocyte subsets are associated with clinical outcomes in severe malarial anaemia and cerebral malaria. Scientific reports $\mathbf{9}(1), 1-13$ (2019)

45. Ortega-Pajares, A., Rogerson, S.J.: The rough guide to monocytes in malaria infection. Frontiers in immunology 9, 2888 (2018)

46. Rizzetto, S., Eltahla, A.A., Lin, P., Bull, R., Lloyd, A.R., Ho, J.W., Venturi, V., Luciani, F.: Impact of sequencing depth and read length on single cell rna sequencing data of t cells. Scientific reports 7(1), 1-11 (2017)

47. Kakani, P., Suman, S., Gupta, L., Kumar, S.: Ambivalent outcomes of cell apoptosis: a barrier or blessing in malaria progression. Frontiers in Microbiology 7, 302 (2016)

48. Penha-Gonçalves, C.: Genetics of malaria inflammatory responses: a pathogenesis perspective. Frontiers in immunology 10, 1771 (2019)

49. Guha, M., Kumar, S., Choubey, V., Maity, P., Bandyopadhyay, U., Guha, M., Kumar, S., Choubey, V., Maity, P., Bandyopadhyay, U.: Apoptosis in liver during malaria: role of oxidative stress and implication of mitochondrial pathway. The FASEB Journal 20(8), 1224-1226 (2006)

50. Bachmann, A., Bruske, E., Krumkamp, R., Turner, L., Wichers, J.S., Petter, M., Held, J., Duffy, M.F., Sim, B.K.L., Hoffman, S.L., et al.: Controlled human malaria infection with plasmodium falciparum demonstrates impact of naturally acquired immunity on virulence gene expression. PLoS pathogens 15(7), 1007906 (2019)

51. Abraham, S., Clark, A.: Dual-specificity phosphatase 1: a critical regulator of innate immune responses. Portland Press Ltd. (2006)

52. Rottenberg, M.E., Carow, B.: Socs3, a major regulator of infection and inflammation. Frontiers in immunology 5, 58 (2014)

53. King, T., Lamb, T.: Interferon- $\gamma$ : the jekyll and hyde of malaria. PLoS pathogens 11(10) (2015) 
54. Ioannidis, L.J., Nie, C.Q., Hansen, D.S.: The role of chemokines in severe malaria: more than meets the eye. Parasitology 141(5), 602-613 (2014)

55. Dunst, J., Kamena, F., Matuschewski, K.: Cytokines and chemokines in cerebral malaria pathogenesis. Frontiers in cellular and infection microbiology 7, 324 (2017)

56. Berg, A., Patel, S., Gonca, M., David, C., Otterdal, K., Ueland, T., Dalen, I., Kvaløy, J.T., Mollnes, T.E., Aukrust, P., et al.: Cytokine network in adults with falciparum malaria and hiv-1: increased il-8 and ip-10 levels are associated with disease severity. PloS one 9(12) (2014)

57. Yoshihama, M., Uechi, T., Asakawa, S., Kawasaki, K., Kato, S., Higa, S., Maeda, N., Minoshima, S., Tanaka, T., Shimizu, N., et al.: The human ribosomal protein genes: sequencing and comparative analysis of 73 genes. Genome research 12(3), 379-390 (2002)

58. Tham, W.-H., Beeson, J.G., Rayner, J.C.: Plasmodium vivax vaccine research-we've only just begun. International journal for parasitology 47(2-3), 111-118 (2017)

59. Barry, A., Waltmann, A., Koepfli, C., Barnadas, C., Mueller, I.: Uncovering the transmission dynamics of Plasmodium vivax using population genetics. Pathog Glob Health. 2015; 109 (3): 142-52. Epub 2015/04/22. https://doi. org/10.1179

60. Chakravorty, S.J., Craig, A.: The role of icam-1 in plasmodium falciparum cytoadherence. European journal of cell biology 84(1), 15-27 (2005)

61. Sobota, R.S., Dara, A., Manning, J.E., Niangaly, A., Bailey, J.A., Kone, A.K., Thera, M.A., Djimdé, A.A., Vernet, G., Leissner, P., et al.: Expression of complement and toll-like receptor pathway genes is associated with malaria severity in mali: a pilot case control study. Malaria journal 15(1), 150 (2016)

62. Abel, A., Steeg, C., Aminkiah, F., Addai-Mensah, O., Addo, M., Gagliani, N., Casar, C., Yar, D.D., Owusu-Dabo, E., Jacobs, T., et al.: Differential expression pattern of co-inhibitory molecules on cd4+ t cells in uncomplicated versus complicated malaria. Scientific reports 8(1), 1-9 (2018)

63. Chaplin, D.D.: Overview of the immune response. Journal of Allergy and Clinical Immunology 125(2), 3-23 (2010)

64. Gallego-Delgado, J., Ty, M., Orengo, J.M., van de Hoef, D., Rodriguez, A.: A surprising role for uric acid: the inflammatory malaria response. Current rheumatology reports 16(2), 401 (2014) 
65. Wang, S., Song, R., Wang, Z., Jing, Z., Wang, S., Ma, J.: S100a8/a9 in inflammation. Frontiers in immunology 9, $1298(2018)$

66. Freihat, L., Wheeler, J.I., Wong, A., Turek, I., Manallack, D., Irving, H.: Irak3 modulates downstream innate immune signalling through its guanylate cyclase activity. Scientific reports 9(1), 1-12 (2019)

67. Hop, H.T., Arayan, L.T., Huy, T.X., Reyes, A.W., Vu, S.H., Min, W., Lee, H.J., Rhee, M.H., Chang, H.H., Kim, S.: The key role of c-fos for immune regulation and bacterial dissemination in brucella infected macrophage. Frontiers in Cellular and Infection Microbiology 8, 287 (2018)

68. Behet, M.C., Kurtovic, L., van Gemert, G.-J., Haukes, C.M., Siebelink-Stoter, R., Graumans, W., van de Vegte-Bolmer, M.G., Scholzen, A., Langereis, J.D., Diavatopoulos, D.A., et al.: The complement system contributes to functional antibody-mediated responses induced by immunization with plasmodium falciparum malaria sporozoites. Infection and immunity 86(7) (2018)

69. Strasser, A., Vaux, D.L.: Viewing bcl2 and cell death control from an evolutionary perspective. Cell Death \& Differentiation 25(1), 13-20 (2018)

70. White, N.J.: Anaemia and malaria. Malaria journal 17(1), 1-17 (2018)

71. Casals-Pascual, C., Kai, O., Cheung, J.O., Williams, S., Lowe, B., Nyanoti, M., Williams, T.N., Maitland, K., Molyneux, M., Newton, C.R., et al.: Suppression of erythropoiesis in malarial anemia is associated with hemozoin in vitro and in vivo. Blood 108(8), 2569-2577 (2006)

72. Lamikanra, A.A., Theron, M., Kooij, T.W., Roberts, D.J.: Hemozoin (malarial pigment) directly promotes apoptosis of erythroid precursors. PloS one 4(12), 8446 (2009)

73. Boulet, C., Doerig, C.D., Carvalho, T.G.: Manipulating eryptosis of human red blood cells: a novel antimalarial strategy? Frontiers in cellular and infection microbiology 8, 419 (2018)

74. Rifaie-Graham, O., Pollard, J., Raccio, S., Balog, S., Rusch, S., Hernández-Castañeda, M.A., Mantel, P.-Y., Beck, H.-P., Bruns, N.: Hemozoin-catalyzed precipitation polymerization as an assay for malaria diagnosis. Nature communications 10(1), 1-8 (2019)

75. Aguilar, R., Moraleda, C., Achtman, A.H., Mayor, A., Quintó, L., Cisteró, P., Nhabomba, A., Macete, E., Schofield, L., Alonso, P.L., et al.: Severity of anaemia is associated with bone marrow haemozoin in children exposed to p lasmodium falciparum. British journal of haematology 164(6), 877-887 (2014) 
76. Chauhan, K., Kalam, H., Dutt, R., Kumar, D.: Rna splicing: A new paradigm in host-pathogen interactions. Journal of molecular biology (2019)

77. Rotival, M., Quach, H., Quintana-Murci, L.: Defining the genetic and evolutionary architecture of alternative splicing in response to infection. Nature communications 10(1), 1-15 (2019)

78. McGuinness, D.H., Dehal, P.K., Pleass, R.J.: Pattern recognition molecules and innate immunity to parasites. Trends in parasitology 19(7), 312-319 (2003)

79. Lübbers, J., Rodríguez, E., Van Kooyk, Y.: Modulation of immune tolerance via siglec-sialic acid interactions. Frontiers in immunology 9, 2807 (2018)

80. Raulf, M.-K., Johannssen, T., Matthiesen, S., Neumann, K., Hachenberg, S., Mayer-Lambertz, S., Steinbeis, F., Hegermann, J., Seeberger, P.H., Baumgärtner, W., et al.: The c-type lectin receptor clec12a recognizes plasmodial hemozoin and contributes to cerebral malaria development. Cell reports 28(1), 30-38 (2019)

81. Maglinao, M., Klopfleisch, R., Seeberger, P.H., Lepenies, B.: The c-type lectin receptor dcir is crucial for the development of experimental cerebral malaria. The Journal of Immunology 191(5), 2551-2559 (2013)

82. White, N.J.: Malaria parasite clearance. Malaria journal 16(1), 88 (2017)

83. Clark, I.A., Budd, A.C., Alleva, L.M., Cowden, W.B.: Human malarial disease: a consequence of inflammatory cytokine release. Malaria journal 5(1), 85 (2006)

84. Gowda, D., Wu, X.: Parasite recognition and signaling mechanisms in innate immune responses to malaria. Frontiers in immunology 9, 3006 (2018)

85. Lemaire, D., Barbosa, T., Rihet, P.: Coping with genetic diversity: the contribution of pathogen and human genomics to modern vaccinology. Brazilian Journal of Medical and Biological Research 45(5), 376-385 (2012)

86. Millwood, I.Y., Bennett, D.A., Walters, R.G., Clarke, R., Waterworth, D., Johnson, T., Chen, Y., Yang, L., Guo, Y., Bian, Z., et al.: A phenome-wide association study of a lipoprotein-associated phospholipase a2 loss-of-function variant in 90000 chinese adults. International journal of epidemiology 45(5), 1588-1599 (2016) 
87. Morris, J.C., Schindler, S.E., McCue, L.M., Moulder, K.L., Benzinger, T.L., Cruchaga, C., Fagan, A.M., Grant, E., Gordon, B.A., Holtzman, D.M., et al.: Assessment of racial disparities in biomarkers for alzheimer disease. JAMA neurology 76(3), 264-273 (2019)

88. Roederer, M.W., McLeod, H., Juliano, J.J.: Can pharmacogenomics improve malaria drug policy? Bulletin of the World Health Organization 89, 838-845 (2011)

\section{Supplementary materials}

Supplementary Figure 1: Globin Gene Removal: Globin Gene Removal in the Yamagishi dataset.

Supplementary Figure 2: Species summary: Principal component analysis of reads mapping to $P$. falciparum and P. vivax.

Supplementary Figure 3: Variance partition: Violin plots of the percentage of variance explained by all covariates used in the linear model.

Supplementary Figure 4: PCA of Island: PCA analysis of island-level variation.

Supplementary Figure 5: Variance between $P$. falciparum and $P$. vivax genes

Supplementary Figure 6: Ribosomal protein genes: Batch-corrected $\log _{2}$ CPM values of ribosomal protein genes, compared between read length and disease status.

Supplementary Table 1 The number of reads at each filtering stage for both datasets.

Supplementary Table 2 Blood proportion estimates calculated by DeconCell [18] and BH-corrected ANOVA p-values of Tukey's HSD post-hoc test on the CLR-transformed blood cell type data.

Supplementary Table 3 Plasmodium stage proportion estimates calculated by CIBERSORT [21].

Supplementary Table 4 Disease status reassignment after assessing the number of reads mapping to Plasmodium species.

Supplementary Table 5 Total number of reads mapping to each Plasmodium gene for each sample. 
Supplementary Table 6 P-values from ANOVA tests between human gene expression levels and each known covariate.

Supplementary Table 7 Enriched Reactome pathways for DE genes in P. falciparum and P. vivaxinfected patients, along with Reactome pathways for DE genes in $P$. falciparum and $P$. vivax-infected patients which are unique to the Indonesian population.

Supplementary Table 8 List of shared genes between P. falciparum and P. vivax-infected patients, along with a list of genes which are shared between $P$. falciparum and $P$. vivax-infected patients from Indonesia, Africa, and South America.

Supplementary Table 9 List of all significant genes at an absolute $\log _{2}$ fold change threshold of one and an adjusted p-value of 0.05 for Plasmodium, as well as absolute $\log _{2}$ fold change and p-values for genes unique to Indonesia within individuals infected with P. falciparum or P. vivax.

Supplementary Table 10 Gene Ontology analysis (Biological Processes) enrichment results. 
A

B

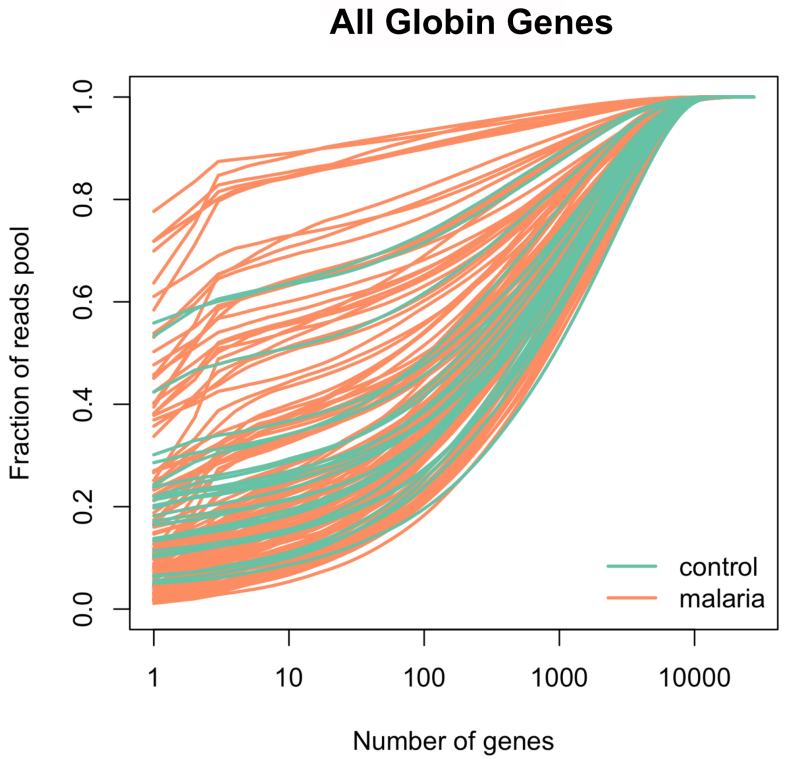

Most Highly Expressed Genes

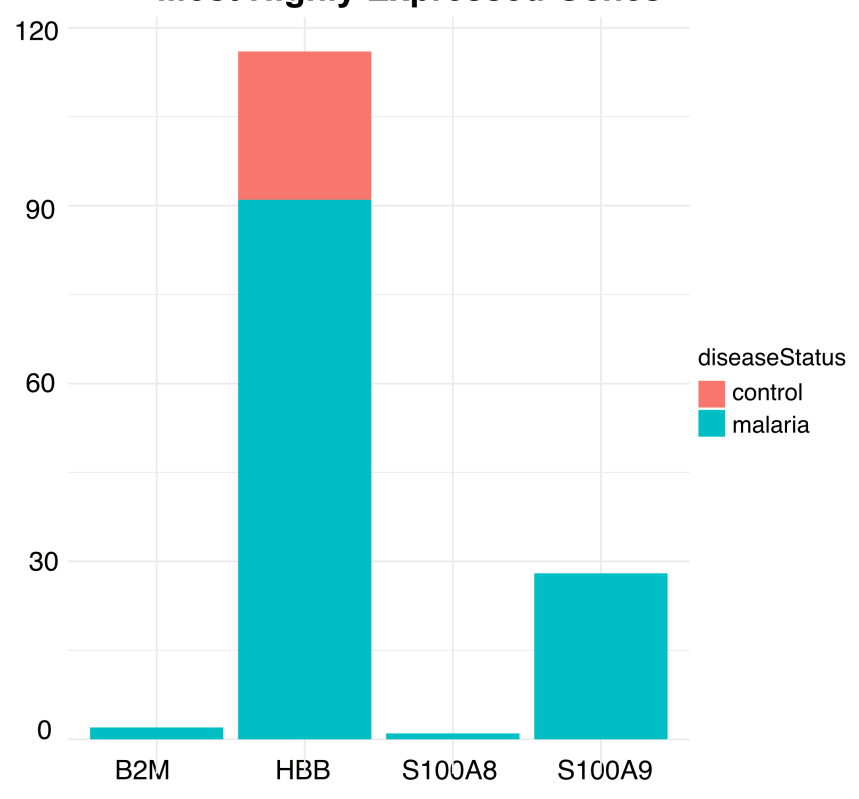

Supplementary Figure 1. Globin gene removal in Yamagishi dataset. A) Most highly expressed genes in the Yamagishi transcriptome blood before removing globin genes. B) Rarefaction curves of the fraction of mRNAs contributed by the number of expressed genes within the Yamagishi dataset before filtering out globin genes. C) Rarefaction curves of the fraction of mRNAs contributed by the number of expressed genes within the Yamagishi dataset after filtering out globin genes. Orange lines indicate samples designated as having malaria by the study authors and green lines indicate samples being classified as healthy controls by the study authors. 

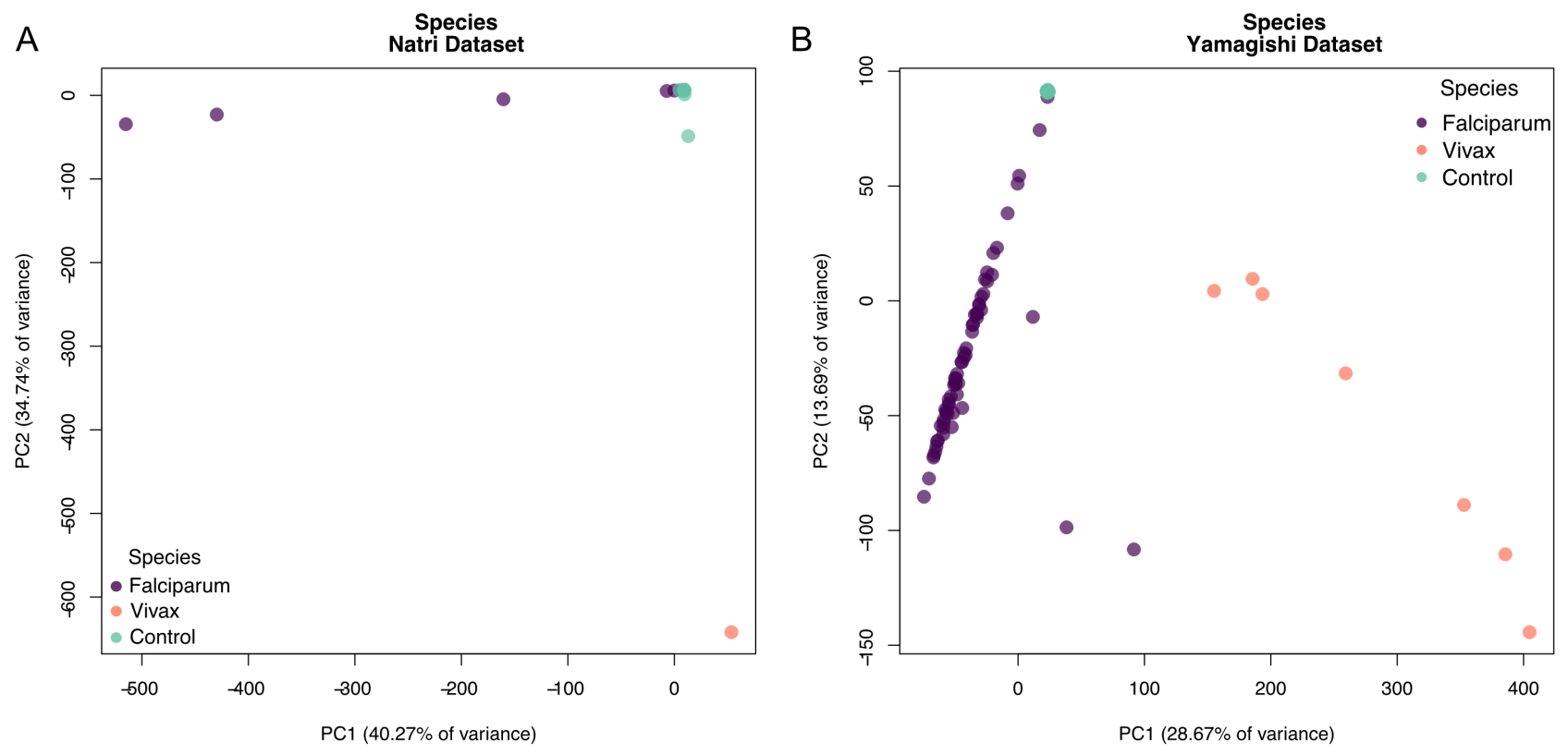

C

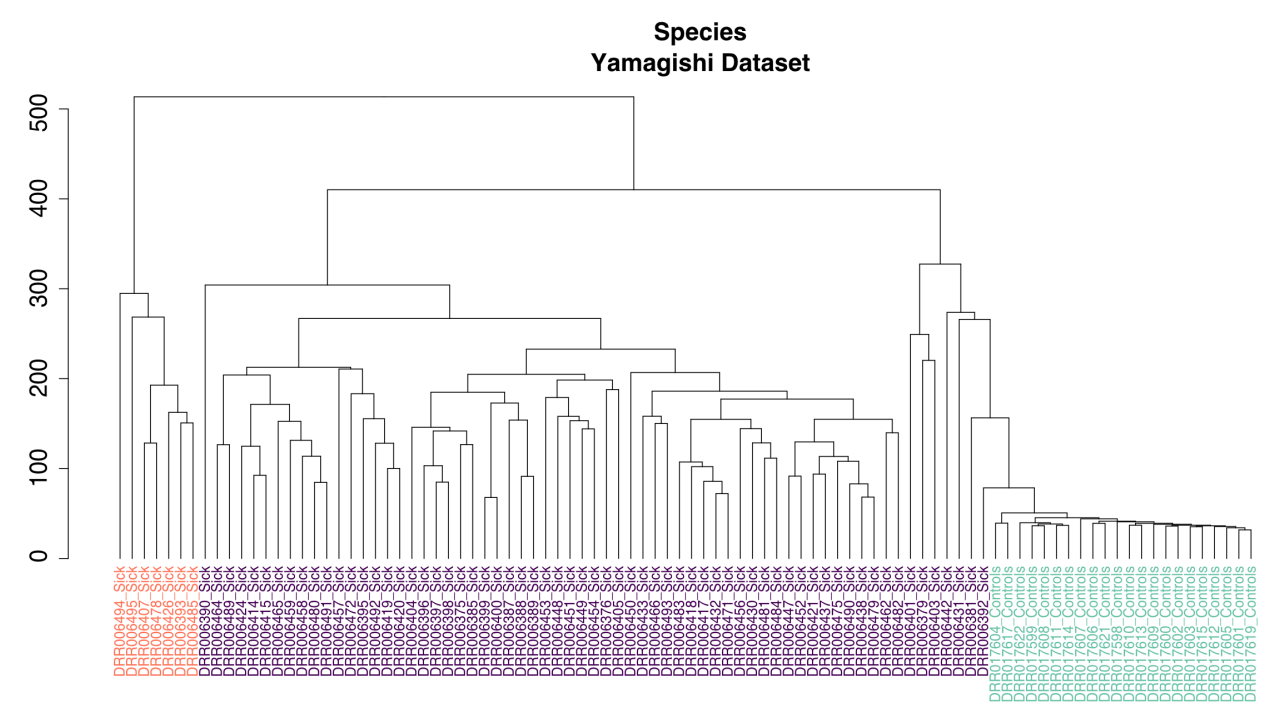

Supplementary Figure 2. Species clustering analysis. A) Principal component analysis of reads mapping to $P$. falciparum and $P$. vivax within the Natri dataset. B) Principal component analysis of reads mapping to $P$. falciparum and $P$. vivax within the Yamagishi dataset. C) Hierarchical clustering by Euclidean distance of reads mapping to $P$. falciparum and $P$. vivax within the Yamagishi dataset. Controls are shown in green, $P$. falciparum in purple, and $P$. vivax in orange. 


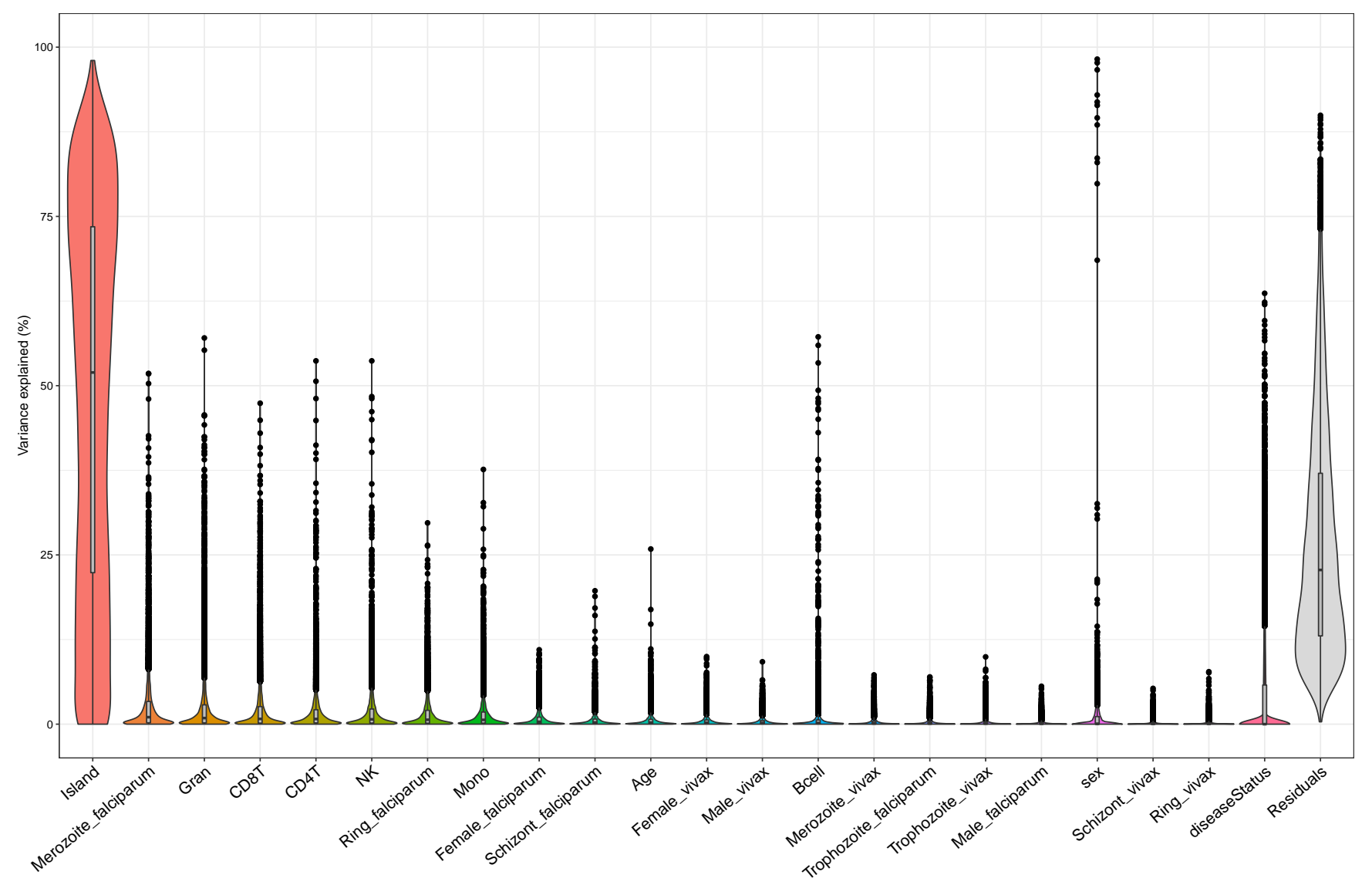

Supplementary Figure 3. Percentage of variance explained by each covariate within the study design. Violin plots of the percentage of variance explained by all covariates used in the linear model. Estimates of variance explained were conducted by the $\mathrm{R}$ package variancePartition. 


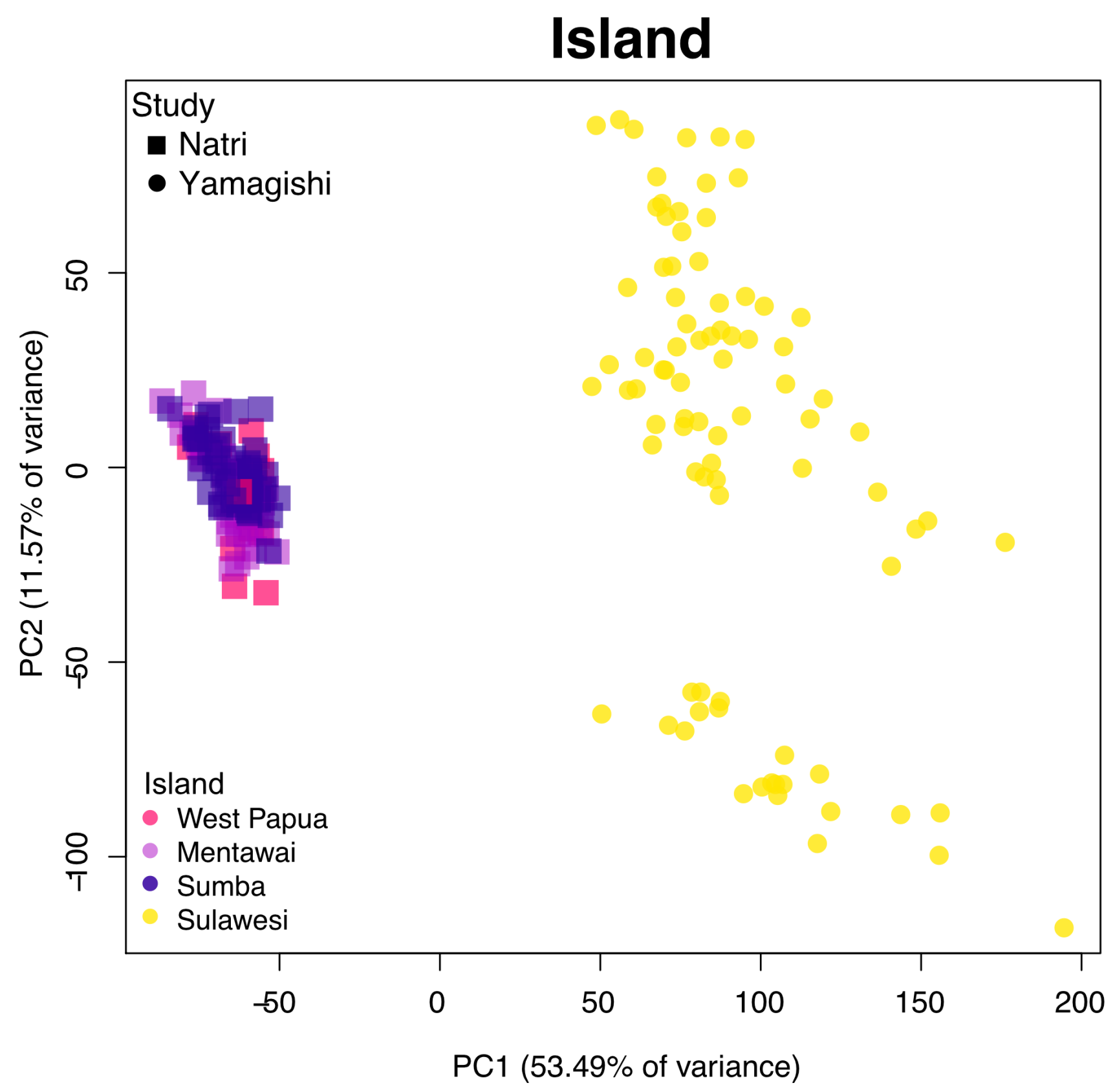

Supplementary Figure 4. PCA analysis of island-level variation before batch correction. 
bioRxiv preprint doi: https://doi.org/10.1101/2021.01.07.425684; this version posted January 8, 2021. The copyright holder for this preprint (which was not certified by peer review) is the author/funder, who has granted bioRxiv a license to display the preprint in perpetuity. It is made available under aCC-BY-NC 4.0 International license.

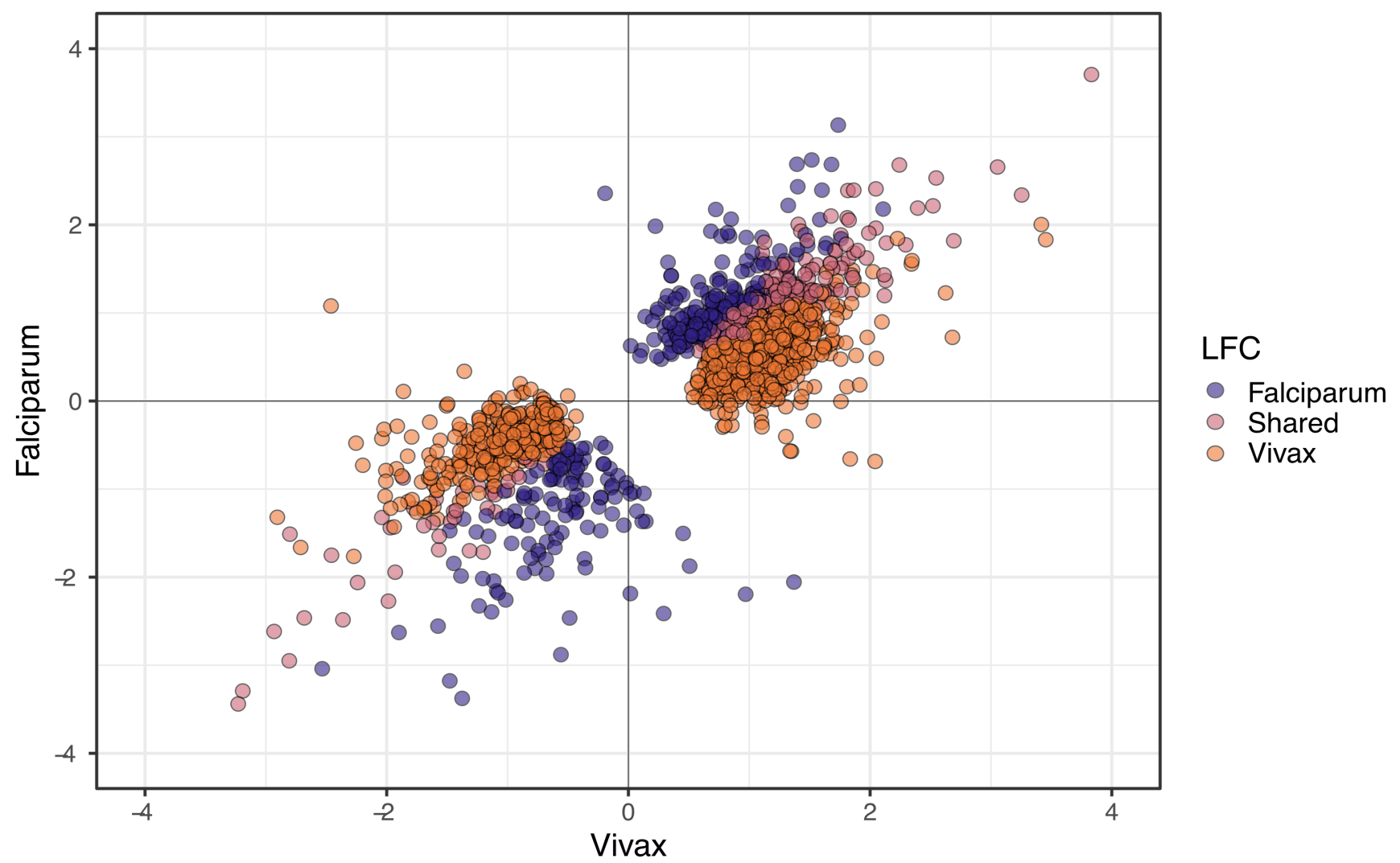

Supplementary Figure 5. Within-gene variance in Indonesian malaria patients. $\log _{2} \mathrm{FC}$ values of significant genes in $P$. falciparum-infected individuals, $P$. vivax-infected individuals, and genes shared between $P$. falciparum and $P$. vivax-infected individuals. 


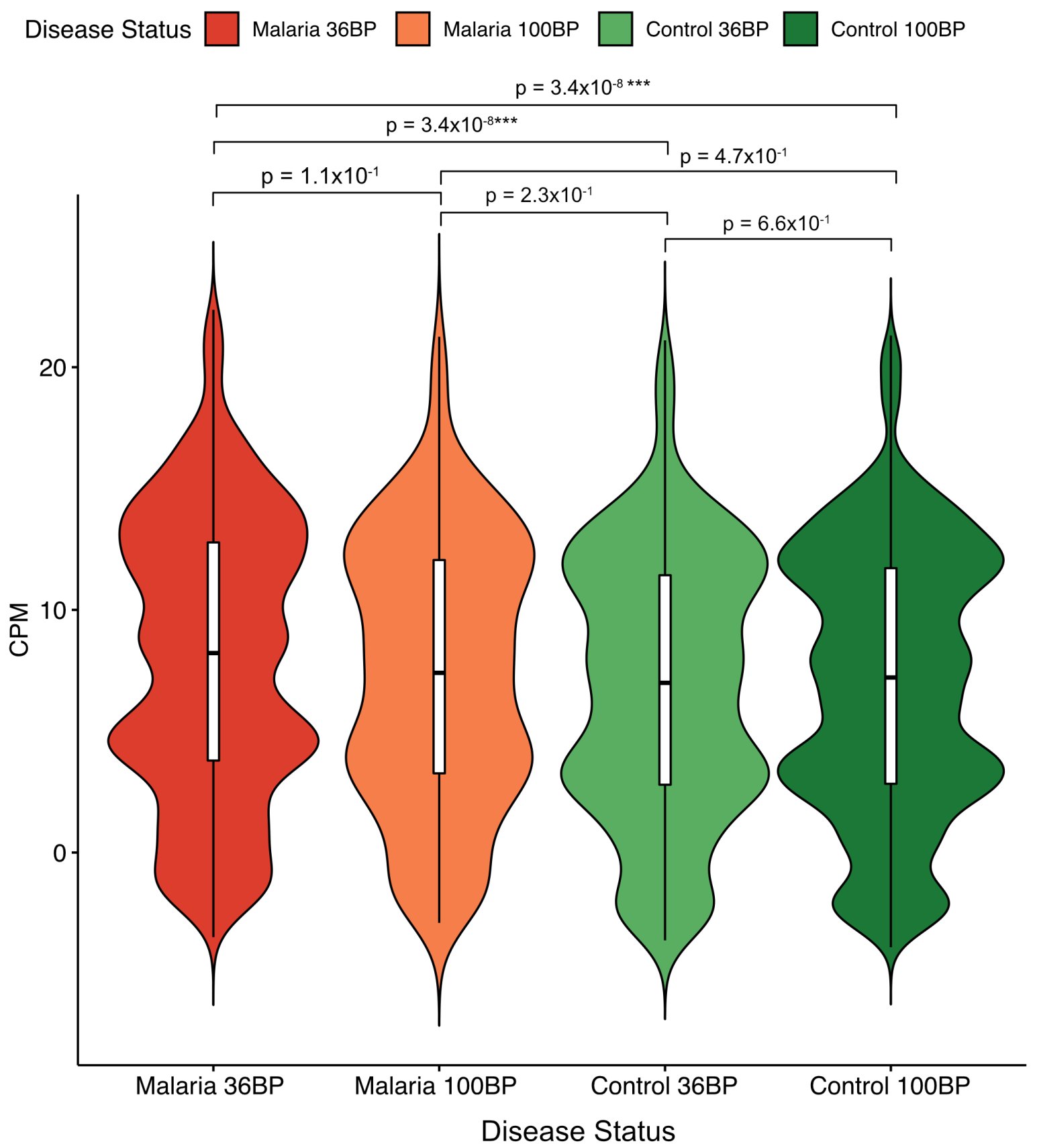

Supplementary Figure 6. Enriched ribosomal protein genes by read length. Batch-corrected $\log _{2}$ CPM values of ribosomal protein genes (genes prefixed with "RPL", "MRPL", and RPS") within cases of 36-BP read length, cases of 100-BP read length, and controls of 36 and 100-BP read length. Adjusted p-values from Tukey's HSD are shown for group comparisons between cases of 36-BP length. 